\title{
Dietary patterns and cardiometabolic risk factors among adolescents: systematic review and meta-analysis
}

\author{
Carla de Magalhães Cunha ${ }^{1,2 *}$, Priscila R. F. Costa ${ }^{3}$, Lucivalda P. M. de Oliveira ${ }^{3}$, \\ Valterlinda A. de O. Queiroz ${ }^{3}$, Jacqueline C. D. Pitangueira ${ }^{1}$ and Ana Marlúcia Oliveira ${ }^{3}$ \\ ${ }^{1}$ Health Science Centre, Federal University of Recôncavo of Babia, Santo Antônio de Jesus, Babia 44574-490, Brazil \\ ${ }^{2}$ Food, Nutrition and Health Post-Graduation Program, Federal University of Bahia, Salvador, Babia 40110-150, Brazil \\ ${ }^{3}$ Science Nutrition Department, Federal University of Babia, Salvador, Babia 40110-150, Brazil
}

(Submitted 15 July 2017 - Final revision received 30 January 2018 - Accepted 31 January 2018)

\section{Abstract}

This study systematised and synthesised the results of observational studies that were aimed at supporting the association between dietary patterns and cardiometabolic risk (CMR) factors among adolescents. Relevant scientific articles were searched in PUBMED, EMBASE, SCIENCE DIRECT, LILACS, WEB OF SCIENCE and SCOPUS. Observational studies that included the measurement of any CMR factor in healthy adolescents and dietary patterns were included. The search strategy retained nineteen articles for qualitative analysis. Among retained articles, the effects of dietary pattern on the means of BMI ( $n$ 18), waist circumference (WC) $(n 9)$, systolic blood pressure ( $n$ 7), diastolic blood pressure $(n 6)$, blood glucose $(n 5)$ and lipid profile $(n 5)$ were examined. Systematised evidence showed that an unhealthy dietary pattern appears to be associated with poor mean values of CMR factors among adolescents. However, evidence of a protective effect of healthier dietary patterns in this group remains unclear. Considering the number of studies with available information, a meta-analysis of anthropometric measures showed that dietary patterns characterised by the highest intake of unhealthy foods resulted in a higher mean BMI $\left(0.57 \mathrm{~kg} / \mathrm{m}^{2} ; 95 \%\right.$ CI $\left.0.51,0.63\right)$ and WC $(0.57 \mathrm{~cm} ; 95 \%$ CI $0.47,0.67)$ compared with low intake of unhealthy foods. Controversially, patterns characterised by a low intake of healthy foods were associated with a lower mean BMI $\left(-0.41 \mathrm{~kg} / \mathrm{m}^{2} ; 95 \%\right.$ CI $\left.-0 \cdot 46,-0 \cdot 36\right)$ and WC $(-0 \cdot 43 \mathrm{~cm}$; $95 \%$ CI $-0.52,-0.33)$. An unhealthy dietary pattern may influence markers of CMR among adolescents, but considering the small number and limitations of the studies included, further studies are warranted to strengthen the evidence of this relation.

\section{Keywords: Dietary patterns: Cardiometabolic risks: Adolescents: Meta-analyses}

The term cardiometabolic risk (CMR) refers to clinical abnormalities that predict chronic disease, such as CVD and/or type 2 diabetes. The term was proposed by the American Diabetes and Heart Associations and has been adopted by scientists and clinicians to describe a set of clinical signs that include hyperinsulinaemia, abdominal obesity, atherogenic dyslipidaemia (reduced HDL-cholesterol and hypertriglyceridaemia) and elevated blood pressure $(\mathrm{BP})^{(1,2)}$

Nowadays, these clinical findings are more frequently diagnosed in youth as a consequence of the worldwide increase in the prevalence of individuals who are overweight or obese ${ }^{(3-6)}$ and as result of unsuccessful strategies to prevent this tendency. Their association with metabolic and vascular abnormalities, the impact on costs to health systems and the low quality of life associated with chronic diseases are concerns among medical societies ${ }^{(7-9)}$. Early exposure to these alterations may contribute to the premature development of atherosclerosis, hypertension and diabetes mellitus in adolescence or during adulthood ${ }^{(10-12)}$.
Considering that adolescents are mainly attracted to unhealthy lifestyles (e.g. sedentary habits, time spent using electronics, meal skipping and a low preference for food with good nutritional value) and are subject to hormonal changes associated with sexual maturation, adolescence can be considered as a vulnerable period of life in terms of $\mathrm{CMR}^{(10,13)}$.

Considering the risk factors associated with lifestyle that adolescents are exposed to, dietary quality is a major contributor and has been considered an important modifiable risk factor for the prevention of cardiometabolic alterations ${ }^{(14)}$. High-quality diets characterised by high intake of fruit, vegetables, legumes, dairy products, whole grains and nuts, which are more evident in prudent/healthy dietary patterns, are associated with protective effects against cardiometabolic diseases among adults ${ }^{(15-17)}$. Among adolescents, such evidence is scarce because few studies have tested the association between dietary patterns and CMR factors and because of the lack of consensus of standardised cut-off points to classify the

Abbreviations: BP, blood pressure; CMR, cardiometabolic risk; DBP, diastolic blood pressure; PC, principal component; SBP, systolic blood pressure; WC, waist circumference.

* Corresponding author: C. de Magalhães Cunha, email carlamagalhaesc@gmail.com 
outcomes in this group, making it difficult to compare studies conducted worldwide ${ }^{(18,19)}$. Therefore, in this investigation, the impact of healthy/unhealthy dietary patterns was evaluated on the mean values of cardiometabolic markers in adolescents. The findings support the lack of consensus in this field through the consolidation of studies that have investigated this relationship. Furthermore, they will contribute to developing intervention programmes and to defining preventive strategies to decrease the progression of CVD in this stage of life.

Thus, the objective of this systematic review and meta-analysis was to systematise and synthesise the results of observational studies with the aim of demonstrating the association between dietary patterns and CMR factors among adolescents.

\section{Methods \\ Search strategy}

From April to June 2017, two researchers independently searched MEDLINE via PubMed, EMBASE, SCIENCE DIRECT, LILACS, WEB OF SCIENCE and SCOPUS. The search criteria were not limited by study publication date, and researchers used the following combination of Medical Subject Heading (MeSH) terms, exploding all trees: 'Dietary Pattern' OR 'Food Pattern' AND 'Blood Pressure' OR 'Diastolic' OR 'Systolic' AND 'Cholesterol' AND 'Blood sugar' OR 'Blood glucose' OR 'Fasting Glucose' AND 'Triglycerides' OR 'Triacylglycerol' AND 'Waist circumference' AND 'Adolescent' OR 'Teen*'.

The search strategy retrieved 4847 articles, and the researchers concluded which studies would be finally selected for data synthesis according to the inclusion and exclusion criteria presented in Table 1. Any disagreement between the researchers during the study selection period was resolved through discussion with a third researcher. The reference lists of the identified papers and thematic reviews were searched, and eleven additional studies were identified. The abstracts from the data sources were pooled and imported into the Endnote Reference Manager in its online version. Fig. 1 summarises the study selection process.

\section{Data extraction and quality assessment}

For each original article included in the final list, the reviewers downloaded and read the entire publication and extracted the following data into a standardised Excel sheet to evaluate quality and to synthesise the evidence: author, year of publication, study's country, sample size, sex, age range, type of study, outcome measurement method, diet assessment method, statistical method used to obtain the food pattern, labels of the identified healthy and unhealthy patterns and the food components, and means and standard deviations of the outcomes of interest.

The results of outcomes presented for the metabolic syndrome, dyslipidaemia, hypertension and insulin resistance were not included considering the variety and low standardisation of cut-off points and references adopted by the scientific community. The classification of healthy and unhealthy patterns was followed according to the authors' descriptions. When two or more healthy or unhealthy patterns were presented, the researcher considered a healthy pattern to mostly include protective foods, such as fruits, legumes, vegetables, lean meats, whole cereals, oils, seeds, tea, natural juice, dairy products and others. An unhealthy pattern mostly consisted of risky foods, such as fast foods, snacks, sugared drinks, candies, transfat and saturated fat sources, fried foods, sugar intake and others. All discrepancies were identified and resolved through discussion, and a third author was included when necessary. Missing data were requested by sending an email to the correspondence author with successful feedback. When outcomes were reported using different measurement units, the units were converted to the most commonly used units in the literature.

The assessment of risk of bias was conducted by two independent reviewers to determine the quality of the included studies. We used a validated reference that was published by Viswanathan \& Berkman $^{(20)}$ to evaluate the risk of bias and precision of observational studies. The Research Triangle Institute (RTI) Item Bank on Risk of Bias and Precision of Observational Studies includes a range of different study designs, and the authors have provided instructions regarding what items to use depending on the studies under assessment. Considering the author recommendation and the type of studies included in this article, we adopted twelve items to evaluate cross-sectional studies (inclusion/exclusion criteria, recruitment strategy, selection of the comparison group, blinding of outcome assessor and validation of measures) and fifteen items for cohort studies (three additional questions about study length

Table 1. Inclusion and exclusion criteria for the selection of observational studies

\begin{tabular}{|c|c|c|}
\hline Criteria & Included & Not included \\
\hline Study type & Observational & Interventional \\
\hline Type of publication & Articles with available full texts & Publications of scientific conference annals \\
\hline Year of publication & All & - \\
\hline Language & English, French, Italian, German, Spanish or Portuguese & Chinese and Arabic \\
\hline Sample characteristics & Samples containing adolescents aged $10-18$ years & $\begin{array}{l}\text { Samples composed only of individuals aged } 9 \text { years or less, } \\
\text { or }>19 \text { years }\end{array}$ \\
\hline & Healthy adolescents & $\begin{array}{l}\text { Adolescents presenting any disease (e.g., diabetes, mental } \\
\text { disturbances, renal disease, hypertension, HIV, cancer, } \\
\text { Down syndrome) }\end{array}$ \\
\hline \multirow[t]{2}{*}{ Exposition } & Dietary patterns as exposition & $\begin{array}{l}\text { Dietary analysis for specific food groups, single nutrients, diet } \\
\text { quality index or meal patterns }\end{array}$ \\
\hline & $\begin{array}{l}\text { Dietary pattern obtained by factorial, principal component, } \\
\text { multiple correspondence and cluster analysis }\end{array}$ & $\begin{array}{l}\text { Cluster or reduced rank regression analysis simultaneously } \\
\text { between diet and physical activity or lifestyle }\end{array}$ \\
\hline Outcome & Cardiometabolic risk results expressed as the means & Cardiometabolic expressed qualitatively \\
\hline
\end{tabular}

,


and the impact of losses during follow-up). It was decided that a cross-sectional study with four or more of the key items being rated negative or unclear could not be rated as low risk of bias. Similarly, cohort studies with five or more negative or unclear items were considered potentially biased studies.

\section{Statistical analysis}

The association between dietary patterns and outcome variables and the respective CI (95\% CI) were quantified by synthesising the data that were extracted using meta-analysis techniques. Random effects models were considered to compare the results between exposure to smaller amounts of food $v$. larger amounts of food that comprised healthy and unhealthy eating patterns because high heterogeneity was observed among selected studies. To obtain the mean differences among the outcomes, the way in which these outcomes were measured was considered. The standardised mean difference (SMD) and weighted mean difference (WMD) were used to evaluate outcomes that were measured following distinct techniques and those obtained by similar methods, respectively ${ }^{(21)}$.

The heterogeneity among the subgroups was quantified and tested with the $I^{2}$ test. To explore the sources of heterogeneity, we evaluated whether the study results differed according to the sample size and mean age of the study population. Considering the reduced number of included studies, the potential publication bias using visual inspection of the funnel chart was not evaluated. Meta-regression analysis was performed on variables that could influence heterogeneity, such as BMI, mean age and sample size of the studies. Energy intake in quintiles/quartiles/tertiles was not used as part of the meta-regression because it was not informed by most of the studies included in the meta-analysis. Furthermore, we undertook the sensibility analysis to investigate the sources of heterogeneity in the meta-analysis. All statistical tests were twosided $(P<0.05)$, and calculations were conducted using the software STATA (StataCorp. 2013, Stata Statistical Software: Release 13; StataCorp LP).

\section{Results}

\section{Study selection}

The search strategy retrieved 4847 articles from the accessed databases. Of these articles, 4194 articles were excluded after duplication screening, and 287 articles were excluded on the basis of title and abstract content. Full-text reading of 366 articles resulted in the exclusion of 347 articles, resulting in nineteen articles for qualitative analysis and seven articles for quantitative analysis. The final number of quantitative studies for each outcome is presented in Fig. 1, considering that a single study may present one or more outcome results of interest for this research.

The selected studies were conducted in Australia ${ }^{(22,23)}$, America $^{(24-28)}$, Asia ${ }^{(29-36)}$, Europe ${ }^{(37-39)}$ and Africa ${ }^{(40)}$. Most were cross-sectional studies, and only two were cohort studies. For diet assessment, eleven studies used a FFQ method (number of items ranged from thirty-eight to 168), and a dietetic pattern was obtained in fourteen studies by using factorial analysis. The characteristics of the retrieved studies and the food content of healthy and unhealthy dietary patterns are described in Tables 2 and 3. In addition, twelve studies were classified as low risk of bias (Table 4).

\section{Dietary patterns and lipid profile}

The association between dietary pattern and serum lipids (total cholesterol, LDL-cholesterol, HDL-cholesterol and TAG) was identified in five studies. In an Australian study, Ambrosini et $a l^{(22)}$ evaluated the association between dietary patterns and the metabolic syndrome in 14-year-old adolescents. They found that higher 'Western' pattern scores obtained by factorial analysis were associated with an increasing trend in total cholesterol ( $P_{\text {for trend }} Z$ 0.03) among girls and that boys showed greater mean HDL-cholesterol concentrations associated with higher 'Healthy' pattern scores ( $P_{\text {for trend }} Z$ 0.02). Ochoa-Avilés et al. ${ }^{(24)}$ identified that 'wheat-dense animal-fat pattern' mainly based on refined wheat products, red meat, animal fat, dairy products and plantain intake with low maize and whole-grain consumption was associated with increased total cholesterol $(P=0.02)$ and LDL-cholesterol $(P=0.04)$ among rural adolescents in Mexico.

In contrast to the positive association of 'Western' dietary pattern with metabolic risk factors, Joung et $a l .{ }^{(32)}$ observed in their data on 3168 Korean adolescents aged 13-18 years old that the 'Rice \& kimchi' pattern, which is similar to a traditional Korean dietary pattern featuring high consumption of white rice, kimchi, beans, vegetables and fish, was related to increased risk of elevated serum TAG (OR 0.45; 95\% CI 0.267, 0.781) and reduced HDL-cholesterol levels (OR 0.684; $95 \%$ CI 0.511, 0.916) compared with a modified traditional pattern and a westernised dietary pattern, respectively. In contrast, another Korean study of children and adolescents conducted by Lee et $a l^{(33)}$ identified that LDL-cholesterol levels were significantly lower in those with a 'Traditional diet' pattern than in those with a 'Mixed diet' pattern $(P<0 \cdot 05)$, but this significant difference disappeared after adjusting for age and sex. No differences in other blood lipid profiles or the atherogenic index among the groups were observed.

In a large-sample cross-sectional study with 5267 Chinese children and adolescents, Shang et al. ${ }^{(34)}$ observed that total cholesterol was significantly higher in those with a 'Healthy' dietary pattern than in those with a 'Western' dietary pattern (4.13 (sD 0.76) v. 4.00 (sD 0.70) $\mathrm{mmol} / \mathrm{l}, P=0.0065)$. However, participants with a 'Western' dietary pattern had significantly higher LDL-cholesterol levels $(2.15 \quad(\mathrm{SD} \quad 0.57) \quad v . \quad 2.07$ (sD 0.64$) \mathrm{mmol} / \mathrm{l}, P=0.0023$ ) and lower HDL-cholesterol levels (1.43 (sD 0.28) v. $1.49(\mathrm{sD} 0 \cdot 30) \mathrm{mmol} / 1, P<0.001)$ compared with those with a 'Healthy' dietary pattern. Participants with a 'Western' dietary pattern also showed significantly higher TAG levels than their counterparts with a 'Healthy' dietary pattern (0.93 (sD 0.45) v. 0.91 (sD 0.48) mmol/l, $P<0.001$ ).

\section{Dietary patterns and serum glucose}

Four cross-sectional studies included in this systematic review tested the influence of dietary patterns on blood glucose level. Shang et al. ${ }^{(34)}$ observed a higher level of fasting glucose among participants with a 'Western' dietary pattern compared with 

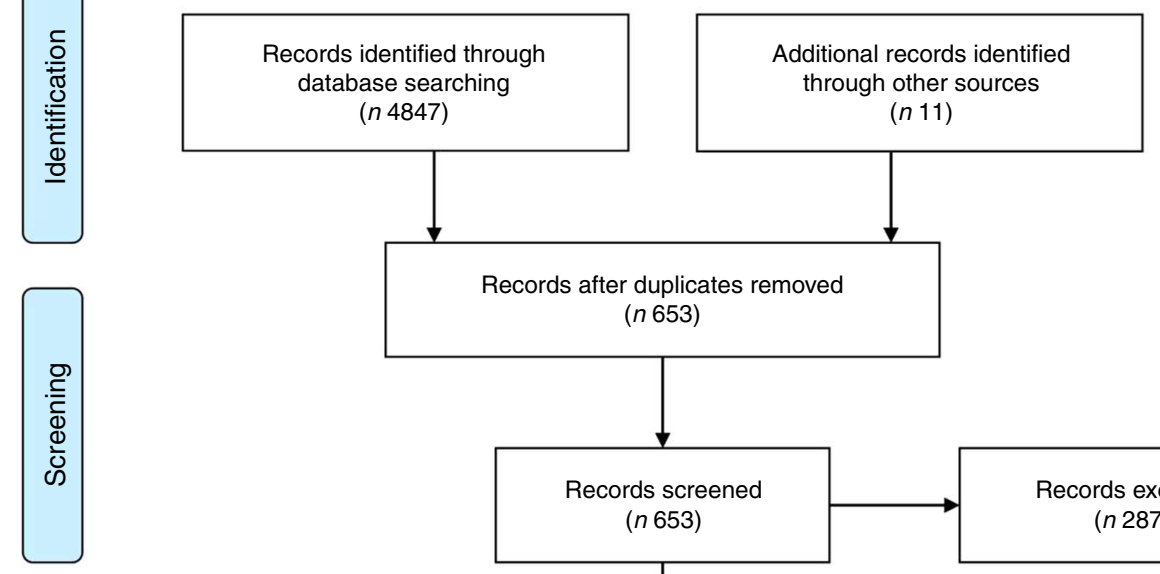
(n 4847)
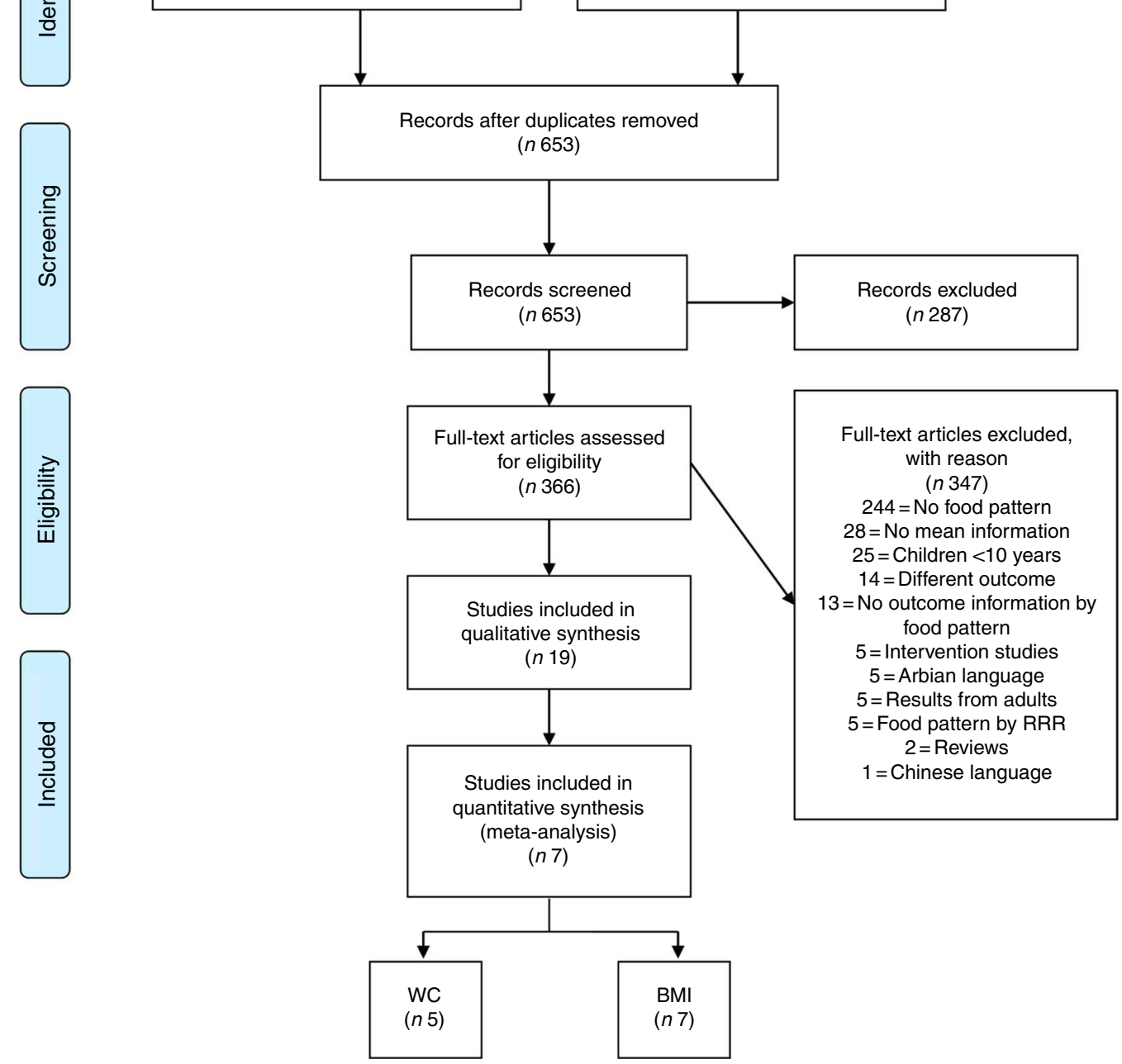

Fig. 1. Flow diagram of the study selection process. RRR, reduced rank regression; WC, waist circumference.

those with a 'Healthy' dietary pattern (4.536 (SD 0.55) v. 4.466 (sD 0.49) $\mathrm{mmol} / \mathrm{l}, P=0 \cdot 0082$ ). Similarly, Ambrosini et $a l^{(22)}$ found that with increasing 'Healthy' dietary pattern scores, mean serum glucose decreased among boys ( $P_{\text {for trend }} Z$ 0.01) and girls ( $P_{\text {for trend }} Z$ 0.04). In a study by Ochoa-Avilés et $a l .{ }^{(24)}$, the 'Ricerich non-animal fat pattern', which reflects a high intake of white rice, vegetable oil and tubers, together with a lower contributions of animal fat, dairy products, pre-packaged food and other cereals in the diet, was correlated with a moderate increase in glucose blood levels among urban Ecuadorian adolescents $(P<0 \cdot 01)$. However, Romero-Polvo et al. ${ }^{(25)}$ found no relationship between mean glucose and highest and low quintiles of dietary patterns in Mexican adolescents aged 10-16 years old.

\section{Dietary patterns and blood pressure}

Measures of BP and its association with dietary patterns were tested by six cross-sectional studies. Hojhabrimanesh et al. ${ }^{(31)}$, in an analysis of a sample of 557 Iranian adolescents, found that the multivariable adjusted means of the systolic BP (SBP) and mean arterial pressure of subjects in the highest tertile of the 'Western' pattern score were significantly higher than those in the lowest tertile (for SBP: mean difference $6.9 \mathrm{mmHg}$, $P=0.001$; and for mean BP: mean difference $4.2 \mathrm{mmHg}$, $P=0.003)$. After stratification for sex and age, the mean values of SBP, diastolic BP (DBP) and mean BP of 12-15-year-old male subjects in the highest tertile of the 'Western' pattern score were significantly higher than those in the lowest tertile (SBP: mean difference $14.5 \mathrm{mmHg}, P=0.001$; for DBP: mean difference $6.5 \mathrm{mmHg}, \quad P=0.015$; and for mean BP: mean difference $9.2 \mathrm{mmHg}, P<0.001)$. A statistically insignificant difference was observed in terms of the multivariable adjusted means of the mean BP of 12-15-year-old girls (mean difference $4.7 \mathrm{mmHg}$, $P=0.064)$.

In the study by McNaughton et al. ${ }^{(23)}$ of adolescents aged more than 16 years, the 'Fruit, salad, cereals and fish' pattern 


\section{N British Journal of Nutrition}

Table 2. Cross-sectional and cohort studies of dietary patterns and cardiometabolic risk markers among adolescents

(Mean values and standard deviations of BMI, waist circumference (WC) and blood pressure (BP))

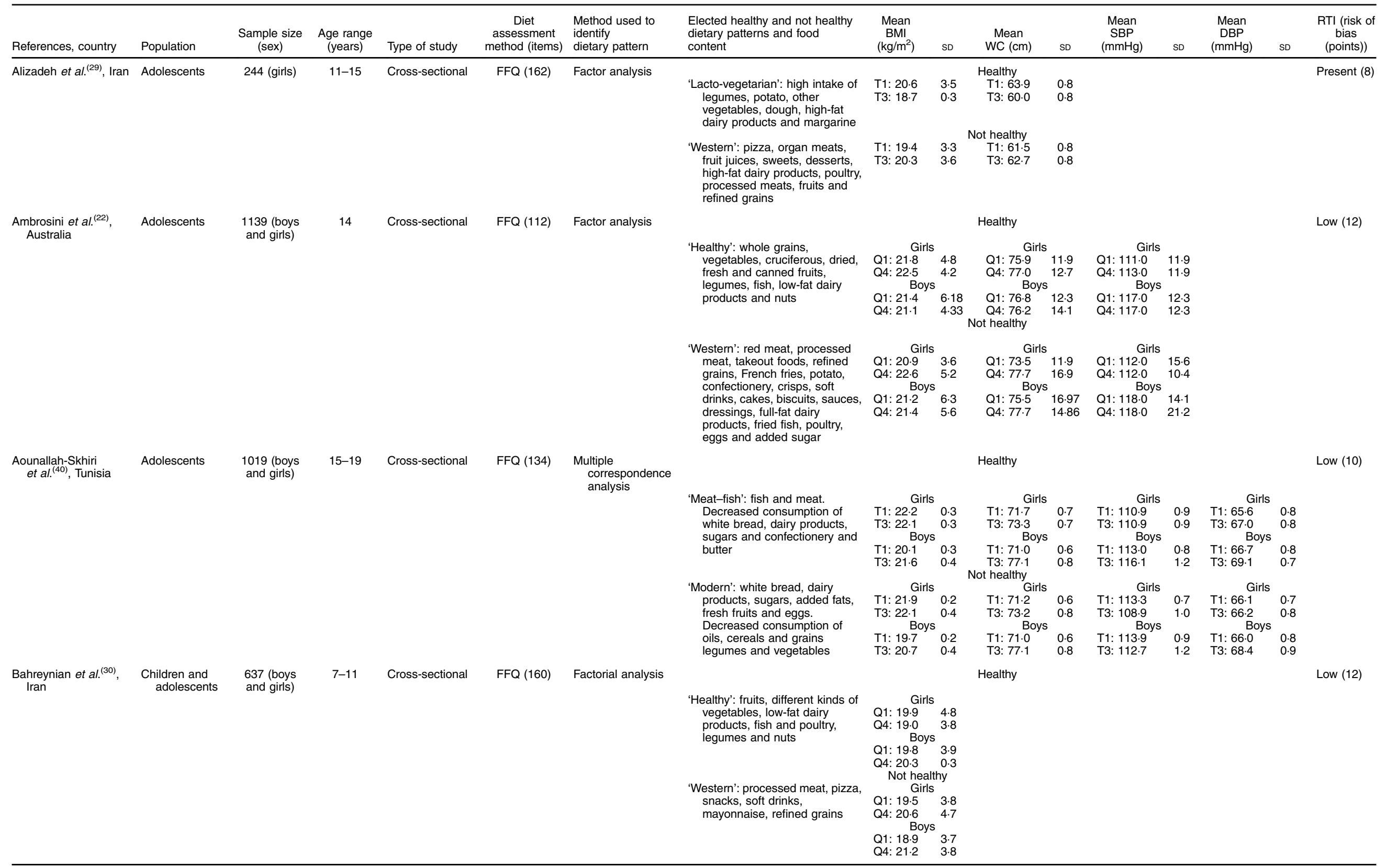




\section{N British Journal of Nutrition}

\section{Table 2. Continued}

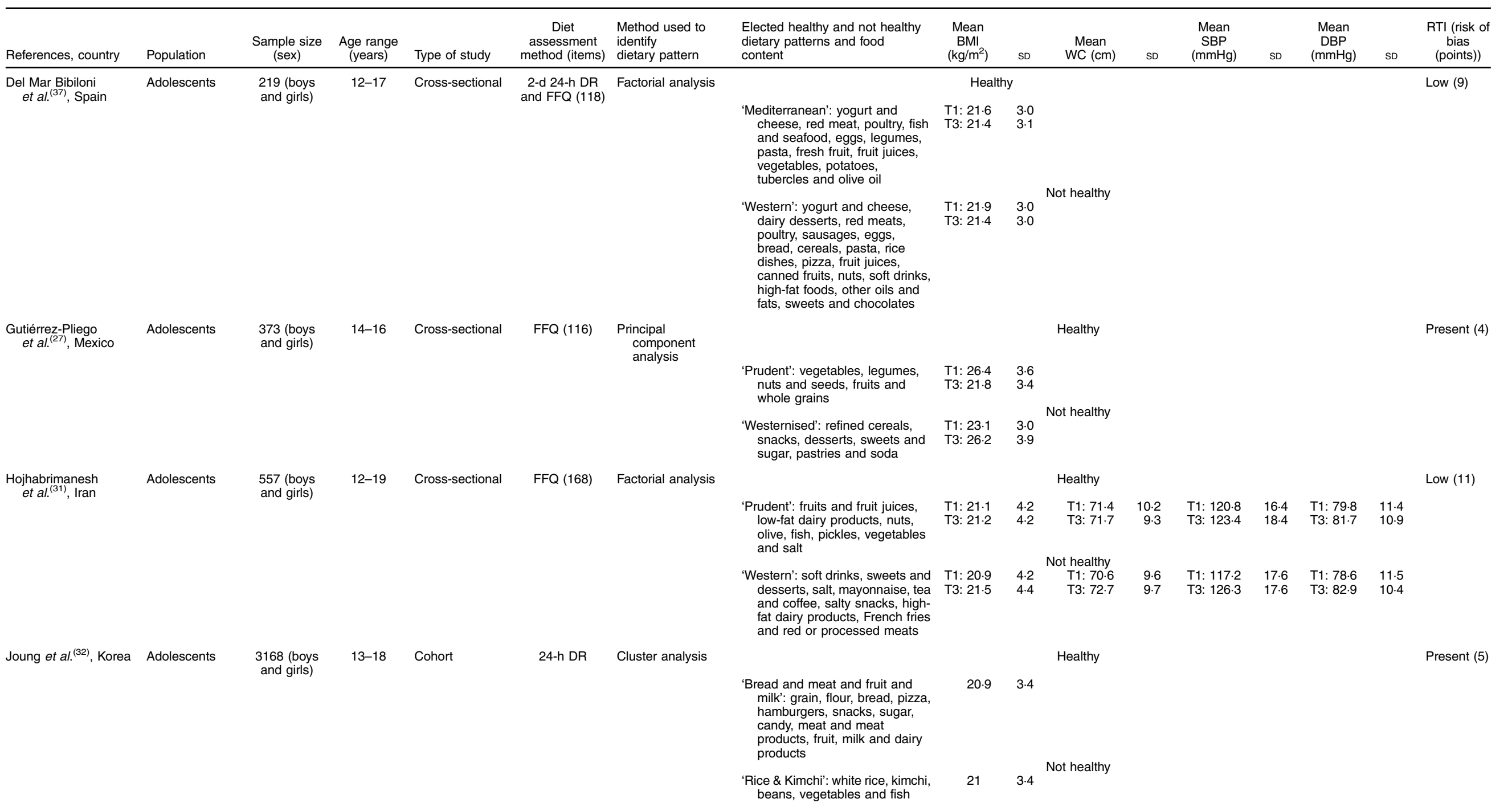




\section{N British Journal of Nutrition}

\section{Table 2. Continued}

\begin{tabular}{|c|c|c|c|c|c|c|c|c|c|c|c|c|c|c|c|c|}
\hline References, country & Population & $\begin{array}{l}\text { Sample size } \\
\text { (sex) }\end{array}$ & $\begin{array}{l}\text { Age range } \\
\text { (years) }\end{array}$ & Type of study & $\begin{array}{c}\text { Diet } \\
\text { assessment } \\
\text { method (items) }\end{array}$ & $\begin{array}{l}\text { Method used to } \\
\text { identify } \\
\text { dietary pattern }\end{array}$ & $\begin{array}{l}\text { Elected healthy and not healthy } \\
\text { dietary patterns and food } \\
\text { content }\end{array}$ & $\begin{array}{c}\text { Mean } \\
\text { BMl } \\
\left(\mathrm{kg} / \mathrm{m}^{2}\right)\end{array}$ & SD & $\begin{array}{l}\text { Mean } \\
\text { WC }(\mathrm{cm})\end{array}$ & SD & $\begin{array}{l}\text { Mean } \\
\text { SBP } \\
(\mathrm{mmHg})\end{array}$ & SD & $\begin{array}{l}\text { Mean } \\
\text { DBP } \\
(\mathrm{mmHg})\end{array}$ & SD & $\begin{array}{c}\text { RTI (risk of } \\
\text { bias } \\
\text { (points)) }\end{array}$ \\
\hline \multirow[t]{3}{*}{ Lee et al. ${ }^{(33)}$, Korea } & $\begin{array}{l}\text { Children and } \\
\text { adolescents }\end{array}$ & $\begin{array}{l}2704 \text { (boys } \\
\text { and girls) }\end{array}$ & $1-19$ & Cross-sectional & 24-h DR & $\begin{array}{l}\text { Factor analysis and } \\
\text { cluster analysis }\end{array}$ & & & & Healthy & & & & & & Present (6) \\
\hline & & & & & & & $\begin{array}{l}\text { 'Traditional': rice, kimchi, fish and } \\
\text { shellfish, beef, vegetables, } \\
\text { seaweeds, oils and oriental } \\
\text { sauces }\end{array}$ & & & & & 111.0 & 11.4 & $65 \cdot 0$ & $10 \cdot 9$ & \\
\hline & & & & & & & $\begin{array}{l}\text { 'Westernised fast food': pizza and } \\
\text { hamburger, poultry, beverages, } \\
\text { cookies, sweets, teas eggs, } \\
\text { poultry, potatoes, processed } \\
\text { meat, western sauce, solid fats } \\
\text { and mayonnaise }\end{array}$ & & & Not healthy & & $110 \cdot 0$ & 13.7 & $65 \cdot 8$ & 10.5 & \\
\hline \multirow{3}{*}{$\begin{array}{l}\text { McNaughton et al. }{ }^{(23)} \text {, } \\
\text { Australia }\end{array}$} & Adolescents & 764 (boys & $12-18$ & Cross-sectional & FFQ (108) & Factorial analysis & & & & Healthy & & & & & & Low (11) \\
\hline & & & & & & & $\begin{array}{l}\text { 'Fruit, salad, cereals and fish': } \\
\text { Fresh fruits, whole grains, } \\
\text { mushrooms, lettuce, tomato, } \\
\text { fish, eggs, oil, nuts and pasta }\end{array}$ & 22.6 & 0.4 & 75.7 & 0.9 & $116 \cdot 1$ & $1 \cdot 2$ & $64 \cdot 3$ & $1 \cdot 1$ & \\
\hline & & & & & & & $\begin{array}{l}\text { 'High fat and sugar': sausage, } \\
\text { biscuits, chocolate, sweet pies, } \\
\text { chips, confectionery, } \\
\text { hamburger, pizza, desserts, } \\
\text { processed meat, flavoured } \\
\text { milk, soft drinks, fried fish, } \\
\text { beef, poultry, pork, ice cream, } \\
\text { fruit juice drink and mince } \\
\text { dishes }\end{array}$ & $22 \cdot 0$ & 0.4 & $\begin{array}{c}\text { Not healthy } \\
74.9\end{array}$ & 0.9 & 118.5 & 1.3 & 67.1 & $1 \cdot 1$ & \\
\hline \multirow{3}{*}{$\begin{array}{l}\text { Monjardino et al. }{ }^{(38)} \text {, } \\
\text { Portugal }\end{array}$} & Adolescents & 1007 & $\begin{array}{l}\text { 13-17 (boys } \\
\text { and girls) }\end{array}$ & Cohort & $\mathrm{FFQ}(91)$ & Cluster analysis & & & & Healthy & & & & & & Low (12) \\
\hline & & & & & & & $\begin{array}{l}\text { 'Healthier': fish, vegetables, } \\
\text { added fats, fruits, pasta/ } \\
\text { potatoes/rice and the lowest } \\
\text { consumption of fast food and } \\
\text { soft drinks }\end{array}$ & $\begin{array}{l}\text { Girls } \\
20 \cdot 5 \\
\text { Boys } \\
20 \cdot 3\end{array}$ & 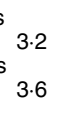 & & & & & & & \\
\hline & & & & & & & $\begin{array}{l}\text { 'Lower intake': significantly lower } \\
\text { consumption of energy, fruits } \\
\text { and of dairy products }\end{array}$ & $\begin{array}{l}\text { Girls } \\
21.5 \\
\text { Boys } \\
21.2\end{array}$ & 5.8 & Not healthy & & & & & & \\
\hline \multirow[t]{3}{*}{$\begin{array}{l}\text { Ochoa-Avilés et al. }{ }^{(24)} \text {, } \\
\text { Ecuador }\end{array}$} & Adolescents & 606 & $\begin{array}{l}10-16 \text { (boys } \\
\text { and girls) }\end{array}$ & Cross-sectional & 2-d 24-h DR & Factor analysis & & & & Healthy & & & & & & Low (12) \\
\hline & & & & & & & $\begin{array}{l}\text { 'Rice-rich non-animal fat': white } \\
\text { rice, vegetable oil and tubers, } \\
\text { lower consumption of animal } \\
\text { fat, dairy products, pre- } \\
\text { packaged food and other } \\
\text { cereals to the diet }\end{array}$ & 20.0 & $2 \cdot 9$ & $70 \cdot 3$ & $7 \cdot 7$ & $101 \cdot 3$ & $10 \cdot 1$ & $62 \cdot 0$ & 8.6 & \\
\hline & & & & & & & $\begin{array}{l}\text { 'Wheat-dense animal fat': refined } \\
\text { wheat products, red meat, } \\
\text { animal fat, dairy products and } \\
\text { plantain intake with low maize } \\
\text { and whole-grain consumption }\end{array}$ & 20.2 & $3 \cdot 1$ & $\begin{array}{c}\text { Not healthy } \\
70 \cdot 3\end{array}$ & 8.1 & 99.0 & $10 \cdot 2$ & 62.5 & 8.9 & \\
\hline
\end{tabular}




\section{N British Journal of Nutrition}

\section{Table 2. Continued}

\begin{tabular}{|c|c|c|c|c|c|c|c|c|c|c|c|c|c|c|c|c|}
\hline References, country & Population & $\begin{array}{l}\text { Sample size } \\
\text { (sex) }\end{array}$ & $\begin{array}{l}\text { Age range } \\
\text { (years) }\end{array}$ & Type of study & $\begin{array}{c}\text { Diet } \\
\text { assessment } \\
\text { method (items) }\end{array}$ & $\begin{array}{l}\text { Method used to } \\
\text { identify } \\
\text { dietary pattern }\end{array}$ & $\begin{array}{l}\text { Elected healthy and not healthy } \\
\text { dietary patterns and food } \\
\text { content }\end{array}$ & $\begin{array}{c}\text { Mean } \\
\text { BMl } \\
\left(\mathrm{kg} / \mathrm{m}^{2}\right)\end{array}$ & SD & $\begin{array}{l}\text { Mean } \\
\text { WC }(\mathrm{cm})\end{array}$ & SD & $\begin{array}{c}\text { Mean } \\
\text { SBP } \\
(\mathrm{mmHg})\end{array}$ & SD & $\begin{array}{l}\text { Mean } \\
\text { DBP } \\
(\mathrm{mmHg})\end{array}$ & SD & $\begin{array}{c}\text { RTI (risk of } \\
\text { bias } \\
\text { (points)) }\end{array}$ \\
\hline \multirow[t]{3}{*}{$\begin{array}{l}\text { Przysławski et al. }{ }^{(39)} \text {, } \\
\text { Poland }\end{array}$} & Adolescents & 479 (girls) & $17-18$ & Cross-sectional & $\begin{array}{l}\text { Self- } \\
\text { administered } \\
\text { dietary } \\
\text { questionnaire }\end{array}$ & Cluster analysis & & & & Healthy & & & & & & Present (4) \\
\hline & & & & & & & $\begin{array}{l}\text { 'Cluster 1': milk and dairy } \\
\text { products, vegetables, fruit and } \\
\text { fish }\end{array}$ & 21.3 & $2 \cdot 8$ & 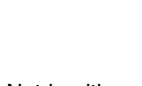 & & & & & & \\
\hline & & & & & & & $\begin{array}{l}\text { 'Cluster 2': lower than average } \\
\text { intake of milk and its products, } \\
\text { vegetables and fruit, more } \\
\text { regular consumption of meals, } \\
\text { especially regular supper } \\
\text { intake }\end{array}$ & 21.8 & 3.2 & Not healthy & & & & & & \\
\hline \multirow[t]{6}{*}{ Ritchie et al. ${ }^{(26)}$, USA } & $\begin{array}{l}\text { Children and } \\
\text { adolescents }\end{array}$ & $\begin{array}{r}2371 \text { (boys } \\
\text { and girls) }\end{array}$ & 9-19 & Cohort & $\begin{array}{l}\text { 3-d 24-h DR } \\
\text { per year }\end{array}$ & $\begin{array}{l}\text { Factor analysis and } \\
\text { cluster analysis }\end{array}$ & & & & $\begin{array}{l}\text { Black girls } \\
\text { Healthy }\end{array}$ & & & & & & Low (14) \\
\hline & & & & & & & $\begin{array}{l}\text { 'Meal-type': plain breads and } \\
\text { grains, other breakfast grains } \\
\text { and most types of sandwiches } \\
\text { and protein sources, including } \\
\text { legumes. Other vegetables } \\
\text { and fried and not fried potatoes }\end{array}$ & 26.8 & 0.3 & 78.5 & 0.5 & & & & & \\
\hline & & & & & & & $\begin{array}{l}\text { 'Sweets and cheese': high } \\
\text { amounts of sweets, flavoured } \\
\text { milk and cheese and small } \\
\text { amounts of eggs, fried fish/ } \\
\text { poultry and fried potatoes }\end{array}$ & $28 \cdot 3$ & $1 \cdot 0$ & $\begin{array}{c}\text { Not healthy } \\
79.7\end{array}$ & 0.3 & & & & & \\
\hline & & & & & & & & & & $\begin{array}{l}\text { White girls } \\
\text { Healthy }\end{array}$ & & & & & & \\
\hline & & & & & & & $\begin{array}{l}\text { 'Healthy': low intake of sweetened } \\
\text { drinks, baked desserts, chips, } \\
\text { fried fish/poultry, red meat, } \\
\text { burgers, pizza and fried } \\
\text { potatoes, and high } \\
\text { consumption of plain milk, } \\
\text { yogurt, plain breads and grains } \\
\text { (without added fats or } \\
\text { condiments), cereal and other } \\
\text { breakfast grains, mixed dishes, } \\
\text { other soups, fruit, green salad, } \\
\text { not fried potatoes and other } \\
\text { vegetables }\end{array}$ & 23.5 & 0.4 & 73.2 & 0.8 & & & & & \\
\hline & & & & & & & $\begin{array}{l}\text { 'Fast food': high in flavoured milk, } \\
\text { burger sandwiches, fried } \\
\text { potatoes, eggs, red meat, } \\
\text { processed meats/ sandwiches, } \\
\text { chips, legumes and baked } \\
\text { desserts. Low in diet drinks, } \\
\text { yogurt, cheese, other desserts, } \\
\text { candy, crackers, pretzels and } \\
\text { peanut butter sandwiches }\end{array}$ & 24.2 & 0.3 & $\begin{array}{c}\text { Not healthy } \\
75.1\end{array}$ & 0.4 & & & & & \\
\hline
\end{tabular}




\section{N British Journal of Nutrition}

\section{Table 2. Continued}

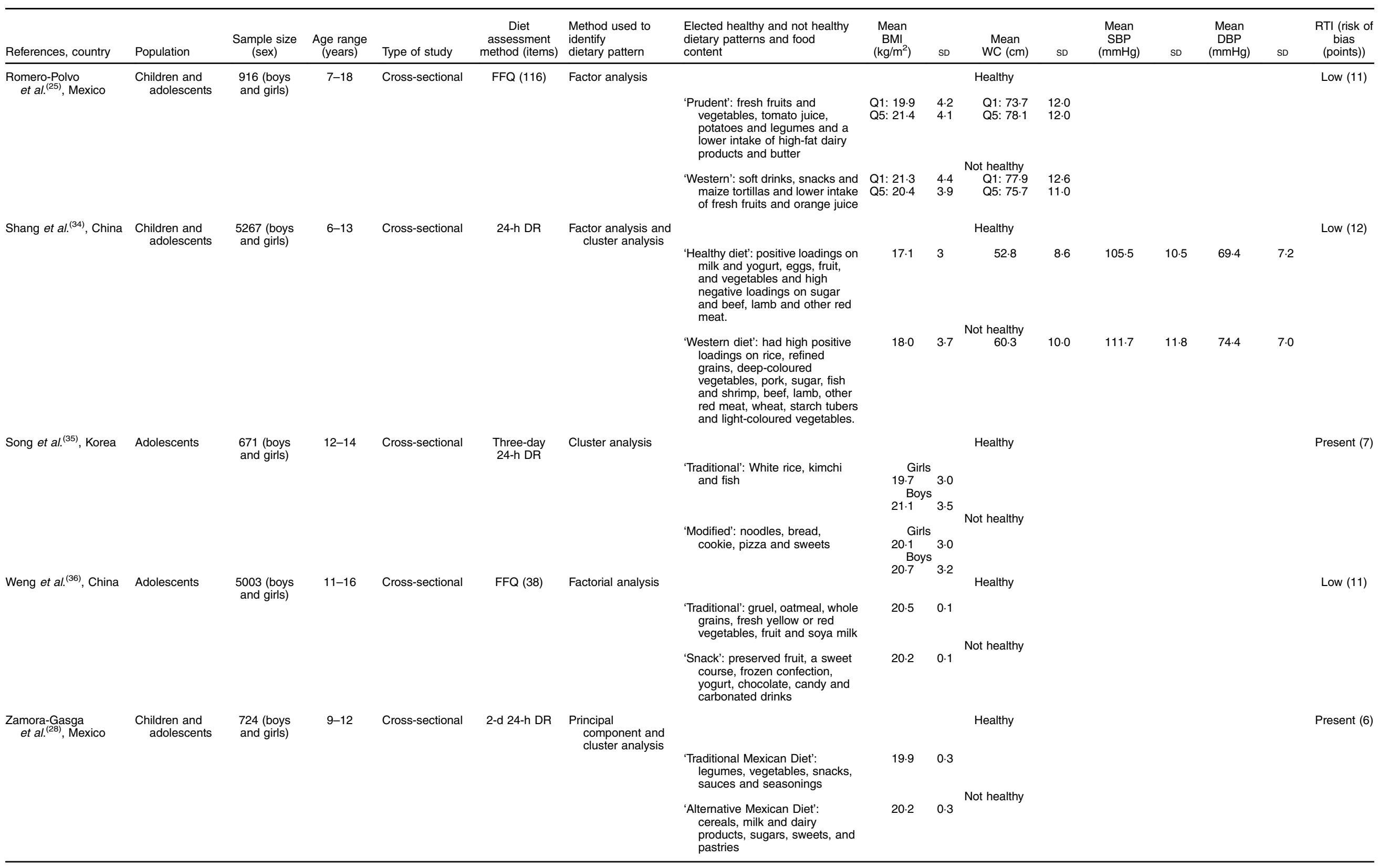

SBP, systolic blood pressure; DBP, diastolic blood pressure; RTI, Research Triangle Institute; Q, quartiles; T, tertiles; DR, dietary record. 


\section{N British Journal of Nutrition}

Table 3. Cross-sectional studies of dietary patterns and cardiometabolic risk markers among adolescents (Mean values and standard deviations of glucose, TAG, LDL and HDL)

\begin{tabular}{|c|c|c|c|c|c|c|c|c|c|c|c|c|c|c|c|c|}
\hline References, country & Population & $\begin{array}{l}\text { Sample size } \\
\text { (sex) }\end{array}$ & $\begin{array}{l}\text { Age range } \\
\text { (years) }\end{array}$ & Type of study & $\begin{array}{c}\text { Diet } \\
\text { assessment } \\
\text { method (items) }\end{array}$ & $\begin{array}{l}\text { Method used to } \\
\text { identify } \\
\text { dietary } \\
\text { pattern }\end{array}$ & $\begin{array}{l}\text { Elected healthy and not } \\
\text { healthy dietary patterns } \\
\text { and food content }\end{array}$ & $\begin{array}{l}\text { Mean } \\
\text { glucose } \\
(\mathrm{mmol} / \mathrm{l})\end{array}$ & SD & $\begin{array}{c}\text { Mean } \\
\text { TAG } \\
(\mathrm{mmol} / /)\end{array}$ & SD & $\begin{array}{c}\text { Mean } \\
\mathrm{HDL} \\
(\mathrm{mmol} / \mathrm{l})\end{array}$ & SD & $\begin{array}{c}\text { Mean } \\
\text { LDL } \\
(\mathrm{mmol} / \mathrm{l})\end{array}$ & SD & $\begin{array}{l}\text { RTI (risk } \\
\text { of bias } \\
\text { (points)) }\end{array}$ \\
\hline \multirow[t]{9}{*}{$\begin{array}{l}\text { Ambrosini et all. } \\
\text { Australia }\end{array}$} & \multirow[t]{9}{*}{ Adolescents } & \multirow[t]{9}{*}{$\begin{array}{l}1139 \text { (boys } \\
\text { and girls) }\end{array}$} & \multirow[t]{9}{*}{14} & \multirow[t]{9}{*}{$\begin{array}{l}\text { Cross- } \\
\text { sectional }\end{array}$} & \multirow[t]{9}{*}{ FFQ (112) } & \multirow[t]{9}{*}{ Factor analysis } & & & & Healthy & & & & & & Low (12) \\
\hline & & & & & & & 'Healthy': whole grains, & Girls & & Girls & & Girls & & $\begin{array}{l}\text { Girls } \\
\end{array}$ & & \\
\hline & & & & & & & $\begin{array}{l}\text { cruciferous, dried, } \\
\text { fresh and canned }\end{array}$ & $\begin{array}{l}\text { T3: } 4.75 \\
\text { Boys }\end{array}$ & 0.65 & Q4: 1.02 & 0.47 & Q4: 1.36 & 0.41 & Q4: 2.53 & 0.83 & \\
\hline & & & & & & & fruits, legumes, fish, & T1: 4.94 & 0.62 & Q1: 0.98 & 0.43 & Q1: 1.25 & 0.37 & Q1: 2.39 & 0.74 & \\
\hline & & & & & & & $\begin{array}{l}\text { low-fat dairy products } \\
\text { and nuts }\end{array}$ & & & Q4: 0.91 & 0.43 & Q4: 1.35 & & Q4: $2 \cdot 43$ & 0.86 & \\
\hline & & & & & & & & & & Not healthy & & & & & & \\
\hline & & & & & & & 'Western': red meat, & Girls & & Girls & & Girls & & Girls & & \\
\hline & & & & & & & $\begin{array}{l}\text { processed meat, } \\
\text { takeaway foods, }\end{array}$ & $\begin{array}{l}\text { T1: } 4 \cdot 87 \\
\text { T3: } 4.77\end{array}$ & $\begin{array}{l}0.57 \\
0.67\end{array}$ & $\begin{array}{l}\text { Q1: } 0.98 \\
\text { Q4: } 1.06\end{array}$ & $\begin{array}{l}0.41 \\
0.57\end{array}$ & $\begin{array}{l}\text { Q1: } 1.38 \\
\text { Q4: } 1.39\end{array}$ & $\begin{array}{l}0.36 \\
0.46\end{array}$ & $\begin{array}{l}\text { Q1: } 2 \cdot 43 \\
\text { Q4: } 2 \cdot 51\end{array}$ & $\begin{array}{l}0.78 \\
0.93\end{array}$ & \\
\hline & & & & & & & $\begin{array}{l}\text { fries, potato, } \\
\text { confectionery, crisps, } \\
\text { soft drinks, cakes, } \\
\text { biscuits, sauces, } \\
\text { dressings, full-fat dairy } \\
\text { products, fried fish, } \\
\text { poultry, eggs and } \\
\text { added sugar }\end{array}$ & $\begin{array}{l}\text { T1: } 4.97 \mathrm{~s} \\
\text { T3: } 4.89\end{array}$ & $\begin{array}{l}0.92 \\
0.84\end{array}$ & $\begin{array}{l}\text { Q1: } 0.98 \\
\text { Q4: } 0.93\end{array}$ & $\begin{array}{l}0.63 \\
0.56\end{array}$ & $\begin{array}{l}\text { Q1: } 1.22 \\
\text { Q4: } 1.30\end{array}$ & $\begin{array}{l}0.56 \\
0.49\end{array}$ & $\begin{array}{l}\text { Q1: } 2 \cdot 34 \\
\text { Q4: } 2 \cdot 36\end{array}$ & $\begin{array}{l}1.20 \\
1.06\end{array}$ & \\
\hline \multirow{3}{*}{$\begin{array}{l}\text { Joung, et al }{ }^{(32)} \text {, } \\
\text { Korea }\end{array}$} & \multirow[t]{3}{*}{ Adolescents } & \multirow{3}{*}{$\begin{array}{l}3168 \text { (boys } \\
\text { and girls) }\end{array}$} & \multirow[t]{3}{*}{$13-18$} & \multirow[t]{3}{*}{ Cohort } & \multirow[t]{3}{*}{ 24-h DR } & \multirow[t]{3}{*}{ Cluster analysis } & & & & Healthy & & & & & & Present (5) \\
\hline & & & & & & & $\begin{array}{l}\text { 'Bread and meat and fruit } \\
\text { and milk': grain, flour, } \\
\text { bread, pizza, } \\
\text { hamburgers, snacks, } \\
\text { sugar, candy, meat } \\
\text { and meat products, } \\
\text { fruit, milk and dairy } \\
\text { products }\end{array}$ & 5.04 & 0.60 & & & 1.31 & 0.30 & & & \\
\hline & & & & & & & $\begin{array}{l}\text { 'Rice and Kimchi': white } \\
\text { rice, kimchi, beans, } \\
\text { vegetables and fish }\end{array}$ & 5.04 & 0.63 & Not healthy & & 1.26 & 0.27 & & & \\
\hline \multirow[t]{3}{*}{ Lee et al. ${ }^{(33)}$, Korea } & \multirow[t]{3}{*}{$\begin{array}{l}\text { Children and } \\
\text { adolescents }\end{array}$} & \multirow[t]{3}{*}{$\begin{array}{l}2704 \text { (boys } \\
\text { and girls) }\end{array}$} & \multirow[t]{3}{*}{$1-19$} & \multirow[t]{3}{*}{$\begin{array}{l}\text { Cross- } \\
\text { sectional }\end{array}$} & \multirow[t]{3}{*}{ 24-h DR } & \multirow[t]{3}{*}{$\begin{array}{l}\text { Factor analysis } \\
\text { and cluster } \\
\text { analysis }\end{array}$} & & & & Healthy & & & & & & Present (6) \\
\hline & & & & & & & $\begin{array}{l}\text { 'Traditional': rice, kimchi, } \\
\text { fish and shellfish, } \\
\text { beef, vegetables, } \\
\text { seaweeds, oils and } \\
\text { oriental sauces }\end{array}$ & & & $1 \cdot 18$ & 0.07 & 1.21 & 0.25 & $2 \cdot 40$ & 0.58 & \\
\hline & & & & & & & $\begin{array}{l}\text { 'Westernised fast food': } \\
\text { pizza and hamburger, } \\
\text { poultry, beverages, } \\
\text { cookies, sweets, teas } \\
\text { eggs, poultry, } \\
\text { potatoes, processed } \\
\text { meat, western sauce, } \\
\text { solid fats and } \\
\text { mayonnaise }\end{array}$ & & & $\begin{array}{l}\text { Not healthy } \\
1.04\end{array}$ & 0.45 & 1.24 & 0.27 & 2.45 & 0.59 & \\
\hline
\end{tabular}




\section{N British Journal of Nutrition}

\section{Table 3. Continued}

\begin{tabular}{|c|c|c|c|c|c|c|c|c|c|c|c|c|c|c|c|c|}
\hline References, country & Population & $\begin{array}{l}\text { Sample size } \\
\text { (sex) }\end{array}$ & $\begin{array}{l}\text { Age range } \\
\text { (years) }\end{array}$ & Type of study & $\begin{array}{c}\text { Diet } \\
\text { assessment } \\
\text { method (items) }\end{array}$ & $\begin{array}{l}\text { Method used to } \\
\text { identify } \\
\text { dietary } \\
\text { pattern }\end{array}$ & $\begin{array}{l}\text { Elected healthy and not } \\
\text { healthy dietary patterns } \\
\text { and food content }\end{array}$ & $\begin{array}{l}\text { Mean } \\
\text { glucose } \\
(\mathrm{mmol} / \mathrm{l})\end{array}$ & SD & $\begin{array}{c}\text { Mean } \\
\text { TAG } \\
(\mathrm{mmol} / \mathrm{l})\end{array}$ & SD & $\begin{array}{c}\text { Mean } \\
\mathrm{HDL} \\
(\mathrm{mmol} / \mathrm{l})\end{array}$ & SD & $\begin{array}{c}\text { Mean } \\
\text { LDL } \\
(\mathrm{mmol} / \mathrm{l})\end{array}$ & SD & $\begin{array}{l}\text { RTI (risk } \\
\text { of bias } \\
\text { (points)) }\end{array}$ \\
\hline \multirow{3}{*}{$\begin{array}{l}\text { Ochoa-Avilés } \\
\text { et al. }{ }^{(24)} \text {, Ecuador }\end{array}$} & Adolescents & 606 (boys and & $10-16$ & Cross- & Two-day 24-h & Factor analysis & & & & Healthy & & & & & & Low (12) \\
\hline & & & & & & & $\begin{array}{l}\text { 'Rice-rich non-animal fat': } \\
\text { white rice, vegetable } \\
\text { oil and tubers, lower } \\
\text { contribution of animal } \\
\text { fat, dairy products, } \\
\text { pre-packaged food } \\
\text { and other cereals to } \\
\text { the diet }\end{array}$ & $4 \cdot 32$ & 0.57 & $1 \cdot 10$ & 0.51 & $1 \cdot 21$ & 0.28 & 1.91 & 0.94 & \\
\hline & & & & & & & $\begin{array}{l}\text { 'Wheat-dense animal- } \\
\text { fat': refined wheat } \\
\text { products, red meat, } \\
\text { animal fat, dairy } \\
\text { products and plantain } \\
\text { intake with low maize } \\
\text { and whole-grain } \\
\text { consumption }\end{array}$ & $4 \cdot 11$ & 0.47 & $\begin{array}{l}\text { Not healthy } \\
1.06\end{array}$ & 0.54 & 1.28 & 0.29 & 1.85 & 1.01 & \\
\hline \multirow[t]{3}{*}{$\begin{array}{l}\text { Romero-Polvo } \\
\text { et al. }{ }^{(25)} \text {, Mexico }\end{array}$} & $\begin{array}{l}\text { Children and } \\
\text { adolescents }\end{array}$ & $\begin{array}{l}916 \text { (boys and } \\
\text { girls) }\end{array}$ & $7-18$ & $\begin{array}{l}\text { Cross- } \\
\text { sectional }\end{array}$ & FFQ (116) & Factor analysis & & & & Healthy & & & & & & Low (11) \\
\hline & & & & & & & $\begin{array}{l}\text { 'Prudent': fresh fruits and } \\
\text { vegetables, tomato } \\
\text { juice, potatoes and } \\
\text { legumes, and a lower } \\
\text { intake of high-fat dairy } \\
\text { products and butter }\end{array}$ & $\begin{array}{l}\text { Q1: } 4.72 \\
\text { Q5: } 4 \cdot 70\end{array}$ & $\begin{array}{l}0.43 \\
0.42\end{array}$ & & & & & & & \\
\hline & & & & & & & $\begin{array}{l}\text { 'Western': soft drinks, } \\
\text { snacks and maize } \\
\text { tortillas and lower } \\
\text { intake of fresh fruits } \\
\text { and orange juice }\end{array}$ & $\begin{array}{l}\text { Q1: } 4.73 \\
\text { Q5: } 4.70\end{array}$ & $\begin{array}{l}0.42 \\
0.44\end{array}$ & Not healthy & & & & & & \\
\hline \multirow[t]{3}{*}{$\begin{array}{l}\text { Shang et al.(34), } \\
\text { China }\end{array}$} & $\begin{array}{l}\text { Children and } \\
\text { adolescents }\end{array}$ & $\begin{array}{l}5267 \text { (boys } \\
\text { and girls) }\end{array}$ & $6-13$ & $\begin{array}{l}\text { Cross- } \\
\text { sectional }\end{array}$ & 24-h DR & $\begin{array}{l}\text { Factor analysis } \\
\text { and cluster } \\
\text { analysis }\end{array}$ & & & & Healthy & & & & & & Low (12) \\
\hline & & & & & & & $\begin{array}{l}\text { 'Healthy diet': positive } \\
\text { loadings on milk and } \\
\text { yogurt, eggs, fruit and } \\
\text { vegetables, and high } \\
\text { negative loadings on } \\
\text { sugar and beef, lamb } \\
\text { and other red meat }\end{array}$ & 4.46 & 0.48 & 0.90 & 0.47 & 1.48 & 0.29 & 2.06 & 0.63 & \\
\hline & & & & & & & $\begin{array}{l}\text { 'Western diet': high } \\
\text { positive loadings on } \\
\text { rice, refined grains, } \\
\text { deep-coloured } \\
\text { vegetables, pork, } \\
\text { sugar, fish and shrimp, } \\
\text { beef, lamb, other red } \\
\text { meat, wheat, starch } \\
\text { tubers and light- } \\
\text { coloured vegetables }\end{array}$ & 4.52 & 0.55 & $\begin{array}{l}\text { Not healthy } \\
0.93\end{array}$ & 0.44 & 1.42 & 0.27 & $2 \cdot 14$ & 0.56 & \\
\hline
\end{tabular}

RTI, Research Triangle Institute; T, tertiles; Q, quartiles; DR, dietary record. 


\section{N British Journal of Nutrition}

Table 4. Research Triangle Institute (RTI) quality assessment of observational studies

\begin{tabular}{|c|c|c|c|c|c|c|c|c|}
\hline \multirow[b]{2}{*}{$\begin{array}{l}\text { RTI questions... } \\
\text { References }\end{array}$} & \multicolumn{3}{|c|}{ Sample definition and selection } & \multirow{2}{*}{$\begin{array}{c}\text { Outcomes } \\
\text { Are the important } \\
\text { outcomes pre- } \\
\text { specified by the } \\
\text { researchers? }\end{array}$} & \multicolumn{2}{|c|}{ Soundness of information } & \multicolumn{2}{|r|}{ Follow-up } \\
\hline & $\begin{array}{l}\text { Are critical } \\
\text { inclusion// } \\
\text { exclusion } \\
\text { criteria clearly } \\
\text { stated? }\end{array}$ & $\begin{array}{l}\text { Are the inclusion/ } \\
\text { exclusion criteria } \\
\text { measured using valid } \\
\text { and reliable } \\
\text { measures? }\end{array}$ & $\begin{array}{l}\text { Was the sample size sufficiently large } \\
\text { to detect a clinically significant } \\
\text { difference of } 5 \% \text { or more in at least } \\
\text { one primary outcome measure? }\end{array}$ & & $\begin{array}{l}\text { Are exposures assessed using } \\
\text { valid and reliable measures, } \\
\text { implemented consistently across } \\
\text { all study participants? }\end{array}$ & $\begin{array}{l}\text { Are outcomes assessed using } \\
\text { valid and reliable measures, } \\
\text { implemented consistently } \\
\text { across all study participants? }\end{array}$ & $\begin{array}{l}\text { Is the length of } \\
\text { follow-up the same } \\
\text { for all groups? }\end{array}$ & $\begin{array}{l}\text { Is the length of time following the } \\
\text { intervention/exposure sufficient to } \\
\text { support the evaluation of primary } \\
\text { outcomes and harms? }\end{array}$ \\
\hline Alizadeh et al. ${ }^{(29)}$ & + & - & - & + & + & + & $\begin{array}{l}\text { Not applicable: } \\
\text { cross-sectional }\end{array}$ & Not applicable: cross-sectional \\
\hline $\begin{array}{l}\text { Ambrosini } \\
\text { et al. }\end{array}$ & + & + & + & + & + & + & $\begin{array}{l}\text { Not applicable: } \\
\text { cross-sectional }\end{array}$ & Not applicable: cross-sectional \\
\hline $\begin{array}{l}\text { Aounallah-Skhiri } \\
\text { et al. }{ }^{(40)}\end{array}$ & Partially & $\begin{array}{l}\text { Cannot determine or } \\
\text { measurement } \\
\text { approach not reported }\end{array}$ & + & + & + & + & $\begin{array}{l}\text { Not applicable: } \\
\text { cross-sectional }\end{array}$ & Not applicable: cross-sectional \\
\hline $\begin{array}{l}\text { Bahreynian } \\
\text { et al. }\end{array}$ & + & + & + & + & + & + & $\begin{array}{l}\text { Not applicable: } \\
\text { cross-sectional }\end{array}$ & Not applicable: cross-sectional \\
\hline $\begin{array}{l}\text { Del Mar Bibiloni } \\
\text { et al. }\end{array}$ & Partially & $\begin{array}{l}\text { Cannot determine or } \\
\text { measurement } \\
\text { approach not reported }\end{array}$ & - & + & + & + & $\begin{array}{l}\text { Not applicable: } \\
\text { cross-sectional }\end{array}$ & Not applicable: cross-sectional \\
\hline $\begin{array}{l}\text { Gutiérrez-Pliego } \\
\text { et al. }\end{array}$ & Partially & $\begin{array}{l}\text { Cannot determine or } \\
\text { measurement } \\
\text { approach not reported }\end{array}$ & - & + & + & + & $\begin{array}{l}\text { Not applicable: } \\
\text { cross-sectional }\end{array}$ & Not applicable: cross-sectional \\
\hline $\begin{array}{l}\text { Hojhabrimanesh } \\
\text { et al }{ }^{(31)}\end{array}$ & + & + & - & + & + & + & $\begin{array}{l}\text { Not applicable: } \\
\text { cross-sectional }\end{array}$ & Not applicable: cross-sectional \\
\hline Joung et al. ${ }^{(32)}$ & Partially & $\begin{array}{l}\text { Cannot determine or } \\
\text { measurement } \\
\text { approach not reported }\end{array}$ & - & + & + & $\begin{array}{l}\text { Cannot determine or } \\
\text { measurement approach not } \\
\text { reported }\end{array}$ & $\begin{array}{l}\text { Not applicable: } \\
\text { cross-sectional }\end{array}$ & Not applicable: cross-sectional \\
\hline Lee et $a .^{(33)}$ & + & + & + & + & + & $\begin{array}{l}\text { Cannot determine or } \\
\text { measurement approach not } \\
\text { reported }\end{array}$ & $\begin{array}{l}\text { Not applicable: } \\
\text { cross-sectional }\end{array}$ & Not applicable: cross-sectional \\
\hline $\begin{array}{l}\text { McNaughton } \\
\text { et al. }\end{array}$ & Partially & $\begin{array}{l}\text { Cannot determine or } \\
\text { measurement } \\
\text { approach not reported }\end{array}$ & + & + & + & + & $\begin{array}{l}\text { Not applicable: } \\
\text { cross-sectional }\end{array}$ & Not applicable: cross-sectional \\
\hline $\begin{array}{l}\text { Monjardino } \\
\text { et al. }{ }^{(38)}\end{array}$ & Partially & $\begin{array}{l}\text { Cannot determine or } \\
\text { measurement } \\
\text { approach not reported }\end{array}$ & + & + & + & + & + & + \\
\hline $\begin{array}{l}\text { Ochoa-Avilés } \\
\text { et al. }{ }^{(24)}\end{array}$ & + & + & + & + & + & + & $\begin{array}{l}\text { Not applicable: } \\
\text { cross-sectional }\end{array}$ & Not applicable: cross-sectional \\
\hline $\begin{array}{l}\text { Przysławski } \\
\text { et al. }{ }^{(39)}\end{array}$ & Partially & $\begin{array}{l}\text { Cannot determine or } \\
\text { measurement } \\
\text { approach not reported }\end{array}$ & - & + & + & + & $\begin{array}{l}\text { Not applicable: } \\
\text { cross-sectional }\end{array}$ & Not applicable: cross-sectional \\
\hline Ritchie et al. ${ }^{(26)}$ & + & + & + & + & + & + & + & + \\
\hline $\begin{array}{l}\text { Romero-Polvo } \\
\text { et al. }\end{array}$ & + & + & + & + & + & + & $\begin{array}{l}\text { Not applicable: } \\
\text { cross-sectional }\end{array}$ & Not applicable: cross-sectional \\
\hline Shang et al. ${ }^{(34)}$ & + & + & + & + & + & + & $\begin{array}{l}\text { Not applicable: } \\
\text { cross-sectional }\end{array}$ & Not applicable: cross-sectional \\
\hline Song et al. ${ }^{(35)}$ & Partially & $\begin{array}{l}\text { Cannot determine or } \\
\text { measurement }\end{array}$ & - & + & + & + & $\begin{array}{l}\text { Not applicable: } \\
\text { cross-sectional }\end{array}$ & Not applicable: cross-sectional \\
\hline Weng et al. ${ }^{(36)}$ & + & + & + & + & + & + & $\begin{array}{l}\text { Not applicable: } \\
\text { cross-sectional }\end{array}$ & Not applicable: cross-sectional \\
\hline $\begin{array}{l}\text { Zamora-Gasga } \\
\text { et al. } \text { al }^{(28)}\end{array}$ & Partially & $\begin{array}{l}\text { Cannot determine or } \\
\text { measurement } \\
\text { approach not reported }\end{array}$ & - & + & + & + & $\begin{array}{l}\text { Not applicable: } \\
\text { cross-sectional }\end{array}$ & Not applicable: cross-sectional \\
\hline
\end{tabular}




\section{NS British Journal of Nutrition}

Table 4. Continued

\begin{tabular}{|c|c|c|c|c|c|c|c|c|}
\hline \multirow[b]{2}{*}{$\begin{array}{l}\text { RTI questions/ } \\
\text { Reference }\end{array}$} & \multicolumn{2}{|c|}{ Analysis comparability } & \multicolumn{3}{|c|}{ Analysis outcome } & \multirow{2}{*}{$\begin{array}{c}\text { Interpretation } \\
\\
\text { Are results } \\
\text { believable taking } \\
\text { study limitations } \\
\text { into consideration? }\end{array}$} & \multirow{2}{*}{$\begin{array}{c}\text { Presentation } \\
\text { and reporting } \\
\text { Are particular } \\
\text { sources of } \\
\text { funding and } \\
\text { supports } \\
\text { related? }\end{array}$} & \multirow[b]{2}{*}{$\begin{array}{l}\text { Total } \\
\text { points }\end{array}$} \\
\hline & $\begin{array}{c}\text { Are confounding and/or effect- } \\
\text { modifying variables assessed using } \\
\text { valid and reliable measures across all } \\
\text { study participants? }\end{array}$ & $\begin{array}{l}\text { Were the important confounding } \\
\text { and effect-modifying variables } \\
\text { taken into account in the design } \\
\text { and/or analysis? }\end{array}$ & $\begin{array}{l}\text { In cases of high loss to } \\
\text { follow-up (or differential } \\
\text { loss to follow-up), is the } \\
\text { impact assessed? }\end{array}$ & $\begin{array}{l}\text { Are any important } \\
\text { primary outcomes } \\
\text { missing from the } \\
\text { results? }\end{array}$ & $\begin{array}{l}\text { Are the statistical methods } \\
\text { used to assess the primary } \\
\text { benefit outcomes appropriate } \\
\text { to the data? }\end{array}$ & & & \\
\hline Alizadeh et al. ${ }^{(29)}$ & - & $\begin{array}{l}\text { Partially: some variables taken } \\
\text { into account }\end{array}$ & $\begin{array}{l}\text { Not applicable: cross- } \\
\text { sectional }\end{array}$ & + & + & + & + & 8 \\
\hline $\begin{array}{l}\text { Ambrosini } \\
\text { et al. }\end{array}$ & + & + & $\begin{array}{l}\text { Not applicable: cross- } \\
\text { sectional }\end{array}$ & + & + & + & + & 12 \\
\hline $\begin{array}{l}\text { Aounallah-Skhiri } \\
\text { et al. } 2011\end{array}$ & + & + & $\begin{array}{l}\text { Not applicable: cross- } \\
\text { sectional }\end{array}$ & + & + & + & + & 10 \\
\hline $\begin{array}{l}\text { Bahreynian } \\
\text { et al. }{ }^{(30)}\end{array}$ & + & + & $\begin{array}{l}\text { Not applicable: cross- } \\
\text { sectional }\end{array}$ & + & + & + & + & 12 \\
\hline $\begin{array}{l}\text { Del Mar Bibiloni } \\
\text { et al. }{ }^{(37)}\end{array}$ & + & + & $\begin{array}{l}\text { Not applicable: cross- } \\
\text { sectional }\end{array}$ & + & + & + & + & 9 \\
\hline $\begin{array}{l}\text { Gutiérrez-Pliego } \\
\text { et al. }\end{array}$ & - & - & $\begin{array}{l}\text { Not applicable: cross- } \\
\text { sectional }\end{array}$ & - & Partially & Partially & + & 4 \\
\hline $\begin{array}{l}\text { Hojhabrimanesh } \\
\text { et al. }{ }^{(31)}\end{array}$ & + & + & $\begin{array}{l}\text { Not applicable: cross- } \\
\text { sectional }\end{array}$ & + & + & + & + & 11 \\
\hline Joung et al. ${ }^{(32)}$ & ? & $\begin{array}{l}\text { Partially: some variables taken } \\
\text { into account }\end{array}$ & $\begin{array}{l}\text { Not applicable: cross- } \\
\text { sectional }\end{array}$ & - & + & + & + & 5 \\
\hline Lee et al. ${ }^{(33)}$ & Source for measures not reported & $\begin{array}{l}\text { Partially: some variables taken } \\
\text { into account }\end{array}$ & $\begin{array}{l}\text { Not applicable: cross- } \\
\text { sectional }\end{array}$ & + & Partially & - & - & 6 \\
\hline $\begin{array}{l}\text { McNaughton } \\
\text { et al. }\end{array}$ & + & + & $\begin{array}{l}\text { Not applicable: cross- } \\
\text { sectional }\end{array}$ & + & + & + & + & 11 \\
\hline $\begin{array}{l}\text { Monjardino } \\
\text { et al. }\end{array}$ & + & + & - & + & + & + & + & 12 \\
\hline $\begin{array}{l}\text { Ochoa-Avilés } \\
\text { et al. }\end{array}$ & + & + & $\begin{array}{l}\text { Not applicable: cross- } \\
\text { sectional }\end{array}$ & + & + & + & + & 12 \\
\hline $\begin{array}{l}\text { Przysławski } \\
\text { et al. }\end{array}$ & - & - & $\begin{array}{l}\text { Not applicable: cross- } \\
\text { sectional }\end{array}$ & + & - & - & - & 4 \\
\hline Ritchie et al. ${ }^{(26)}$ & + & + & $\begin{array}{l}\text { Loss to follow-up was } \\
\text { not considered to be } \\
\text { high }\end{array}$ & + & Partially & + & + & 14 \\
\hline $\begin{array}{l}\text { Romero-Polvo } \\
\text { et al. }\end{array}$ & + & + & $\begin{array}{l}\text { Not applicable: cross- } \\
\text { sectional }\end{array}$ & + & + & + & - & 11 \\
\hline Shang et al. ${ }^{(34)}$ & + & + & $\begin{array}{l}\text { Not applicable: cross- } \\
\text { sectional }\end{array}$ & + & + & + & + & 12 \\
\hline Song et al. ${ }^{(35)}$ & - & $\begin{array}{l}\text { Partially: some variables taken } \\
\text { into account }\end{array}$ & $\begin{array}{l}\text { Not applicable: cross- } \\
\text { sectional }\end{array}$ & + & + & + & + & 7 \\
\hline Weng et al. ${ }^{(36)}$ & - & + & $\begin{array}{l}\text { Not applicable: cross- } \\
\text { sectional }\end{array}$ & + & + & + & + & 11 \\
\hline $\begin{array}{l}\text { Zamora-Gasga } \\
\text { et al. }\end{array}$ & - & - & $\begin{array}{l}\text { Not applicable: cross- } \\
\text { sectional }\end{array}$ & + & - & + & + & 6 \\
\hline
\end{tabular}


was significantly associated with $\mathrm{DBP}(P=0.025)$, with participants with higher scores having lower BP (adjusted for age, sex and physical activity). There were no significant associations between any of the dietary patterns and SBP after adjustment for confounders. Significantly higher SBP and DBP were observed by Shang et al. ${ }^{(34)}$ in Chinese children and adolescents with a 'Western' dietary pattern than among children with a 'Healthy' dietary pattern (SBP: 111.76 (SD 11.8) v. 105.561 (SD 0.5) $\mathrm{mmHg}, P=0.04$ and DBP: 74.46 (SD 7.0) $v .69 .46$ (sD 7.2) $\mathrm{mmHg}, P=0.0435$ ). Opposite results were observed by Aounallah-Skhiri et al. ${ }^{(40)}$ In their Tunisian study, girls exhibited decreased SBP with increasing tertiles of the 'Modern' dietary pattern score. In this same group, there was a decrease in the prevalence of high $\mathrm{BP}$ with modernisation of the diet (2nd $v$. 1st tertile adjusted prevalence OR (POR) 0.6; $95 \%$ CI 0.3, 1.0, 3rd $v$. 1st tertile POR $0 \cdot 4 ; 95 \%$ CI $0 \cdot 2,0 \cdot 8)$; this association remained significant but was reduced when adjusted for age, total energy intake or physical activity measures. Among boys, no straightforward associations were observed between BP and the modern diet score or for the 'Meat-fish' diet score after adjusting for energy intake, BMI and waist circumference (WC), and the difference between tertiles on DBP disappeared. A lower prevalence of hypertension in the highest tertile of the 'Modern' dietary pattern was also observed for both genders. In the studies of Lee et $a l .{ }^{(33)}$ and Ambrosini et $a l .{ }^{(22)}$, BP measures did not show significant differences among the clusters or dietary patterns.

\section{Dietary pattern and $B M I$ and waist circumference}

In all, sixteen studies retained in this systematic review evaluated the effect of dietary pattern on anthropometric measures such as BMI and WC. Among the total studies, four studies observed no significant differences in BMI, WC and overweight prevalence and obesity prevalence among tertiles of those with a westernised dietary pattern compared with healthier dietary patterns and clusters $(23,35,37,39)$

Evidence of the relation between diet and anthropometric measures was observed in a study conducted in Iran by Alizadeh et $a l .{ }^{(29)}$. Subjects of the top tertile of the 'Iranian Central Obesity Making Dietary Pattern' (composed by cruciferous vegetables, green leafy vegetables, soft drinks, tomatoes, other vegetables and vegetable oils) group had higher WC than subjects in the lowest tertile $(P=0.03)$. Adjusting for age increased the positive associations of this dietary pattern with WC $(P=0.009)$ and weight $(P=0.04)$. Compared with subjects of the lowest tertile, those in the upper tertile of the 'Lacto Vegetarian Dietary Pattern' (characterised by legumes, potato, other vegetables, dough, high-fat dairy products and margarine) group had significantly lower weight, WC and BMI either before or after controlling for age $(P<0.01)$. In an Australian study ${ }^{(22)}$, girls in the higher quartiles of the 'Western' dietary pattern showed increases in mean WC ( $P_{\text {for }}$ trend $Z$ 0.03) and BMI ( $P_{\text {for trend }} Z$ 0.02). For boys, no relationships were found in mean WC and BMI with dietary pattern. Aounallah-Skhiri et al. ${ }^{(40)}$ observed associations between increased 'Modern' dietary pattern scores and higher means of BMI and WC in males. These associations were minimally confounded by age, total energy intake or physical activity measures. However, males in the 3rd tertile of healthier 'Meat-fish' dietary pattern scores also presented higher mean BMI and WC, either adjusted for confounders. No obvious relationship was observed for this pattern in females. In females, the only association observed with anthropometry was with abdominal fat accumulation, with those in the 3rd $v$. 1st tertile of the 'Modern' score featuring a slightly higher mean WC in the unadjusted analysis, but the strength of the association was further reduced in adjusted analysis.

Similar results were evidenced by Gutiérrez-Pliego et al. ${ }^{(27)}$. Participants in the highest tertiles of the 'Westernised' pattern and the 'High in protein/fat' pattern showed higher BMI compared with those in the highest tertile of the 'Prudent' pattern. Pearson's correlation analysis between BMI and the different dietary pattern scores showed a positive correlation with the 'Westernised' pattern $(r 0.316 ; P<0.01)$ and the 'High in protein/fat' pattern $(r 307 P<0 \cdot 01)$. In contrast, a negative correlation was found for the prudent dietary pattern $(r-576$ $P<0 \cdot 01)$. Hojhabrimanesh et al. ${ }^{(31)}$ also observed a significantly higher mean WC among individuals in the highest tertile of the Western pattern.

In evaluating the association of obesity and dietary patterns, Shang et $a l^{(34)}$ found that children and adolescents with the 'Western' dietary pattern (high positive loadings on beef/lamb/ other red meat, wheat, starch tubers and light-coloured vegetables) had a significantly higher odds of obesity (OR 2.04; $95 \%$ CI $1.38,3.02$ ) compared with those who followed the 'Healthy' dietary pattern (high positive loadings on milk and yogurt, eggs, fruit and vegetables, and high negative loadings on sugar and beef, lamb and other red meat). After adjustment, the association between the 'Western' dietary pattern and obesity was attenuated but still significant (OR 1.79; 95\% CI 1.20, 2.67). Compared with children who followed the 'Healthy' dietary pattern, the fully adjusted OR was 1.80 (95\% CI $1 \cdot 15$, 2.81) for children with the Western dietary pattern after adjusting for confounding factors. Significantly higher odds of abdominal obesity were observed among children and adolescents with the 'Transitive' dietary pattern (high positive loadings on organ meat, pork, seafood, processed meat, edible fungi and algae and light vegetables) and with the 'Western' dietary pattern compared with the 'Healthy' dietary pattern after adjustment (OR 1.35; 95\% CI 1.14, 1.60 for the 'Transitive' dietary pattern and 1.64; 95\% CI 1.14, 2.35 for the 'Western' dietary pattern).

Weng et al. ${ }^{(36)}$ observed that a higher consumption of the 'Snack' dietary pattern (composed mainly of preserved fruit, a sweet course, frozen confection, yogurt, chocolate, candy and carbonated drinks) was associated with age- and sex-adjusted lower BMI and waist:height ratio (WHtR). An increased frequency of consuming the 'Animal' dietary pattern (red meat, organ meat, processed meat, fried meat and other Chinese meat dishes) was generally associated with age- and sex-adjusted lower BMI and WHtR.

A cohort of American girls ${ }^{(26)}$ stratified by race reported at the end of the study that black girls following the 'Sweets and cheese' pattern tended to have the largest values of adiposity measures $(P=0.095$ for percent body fat for the 'Sweets and cheese' $v$. 'Snack-type foods' patterns) and that black girls following the 'Meal-type' pattern tended to have the lowest 
values $(P=0.074$ for WC for the 'Meal-type' $v$. the 'Customary' pattern) after adjusting for potential confounders. Among white girls, those following the 'Healthy' pattern exhibited significantly smaller mean values for WC at the final follow-up and smaller changes in WC (study year 10 to year 1 ) compared with the 'Sweets and snack-type foods' pattern $(P=0.037)$. Girls following the 'Healthy' pattern also tended to have lower body fat $(P=0.063$ for percent body fat for the 'Healthy' $v$. 'Fast food' patterns) after adjusting for potential confounders.

In a study by Zamora-Gasga et al. ${ }^{(28)}$, principal component (PC) scores were used in multiple regression and cluster analysis. The PC corresponding to vegetables, fish and seafood consumption was positively associated with weight (standardised $\beta$-coefficient $=0.124, P<0 \cdot 01$ ) and BMI (standardised $\beta$-coefficient $=0.117, P<0 \cdot 01$ ). The PC characterised by high consumption of legumes and snacks and low intake of beverages was negatively associated with weight (standardised $\beta$-coefficient $=-0.077, P=0.04$ ) and with BMI (standardised $\beta$-coefficient $=-0.073, \quad P=0.04)$. PC corresponding to high consumption of beverages and snacks and low intake of egg was negatively associated with BMI (standardised $\beta$-coefficient $=-0.064 P=0.01$ )

Different results were evidenced by Bahreynian et al. ${ }^{(30)}$ in which girls in the highest quartile and the second quartile of the 'Healthy' pattern were more likely to have higher BMI (OR 2.23; $95 \%$ CI 1.003, 4.96). Girls in the second quartile of the 'Western' dietary pattern had significantly lower BMI than those in the fourth quartile $(\mathrm{OR}=0.46 ; 95 \% \mathrm{CI} 0.21,1.01)$. There was no significant relationship between the 'Western' pattern with BMI among boys; however, a significant association was observed between the first and fourth quartiles of the 'Healthy' pattern $(\mathrm{OR}=0.36 ; 95 \% \mathrm{CI} 0 \cdot 15,0 \cdot 84)$, but the trend was not statistically significant $\left(P_{\text {for }}\right.$ trend $\left.=0.561\right)$. Lee et $a l .{ }^{(33)}$ also identified contradictory results: in their study, the 'Traditional diet' group had the highest obesity rate of $16.4 \%$. However, when the association was analysed within each age group, significant differences had not appeared, but the 'Traditional diet' tended to have higher obesity rates than the other two patterns. RomeroPolvo et $a l^{(25)}$ found that participants presented statistically significant reduced means in BMI, body fat proportion and WC in the highest quintile of the 'Western' pattern and the 'Prudent' pattern.

From the nineteen studies retrieved in the systematic review that considered unhealthy food patterns and CMR factors among adolescents, only seven studies ${ }^{(22,25,27,29,31,37,40)}$ presented outcome information stratified by quintiles/quartiles/ terciles. Among these, only anthropometric measures of BMI and $\mathrm{WC}$ were included in a sufficient number of studies for the meta-analysis. The meta-analysis could not be performed for the other CMR factors, considering the lack of information in reported means or the very few number of studies.

\section{A meta-analysis of unhealthy dietary patterns and cardiometabolic risk factors}

Associations of eating high amounts of unhealthy foods with BMI and WC are shown in Fig. 2. The mean BMI was $0.57 \mathrm{~kg} / \mathrm{m}^{2}$ higher (95\% CI 0.51, 0.63; $\left.P_{\text {heterogeneity }}<0.001 ; I^{2}=96.5 \%\right)$ among those who had a greater intake of unhealthy foods. The association of unhealthy food patterns and WC was higher for the subgroup analysis when only studies that exclusively included adolescents aged 14 years or older (SMD:1.13; $95 \%$ CI $\left.0.98,1.28 ; P_{\text {heterogeneity }}<0.001 ; I^{2}=99.5 \%\right)$ were analysed and compared with samples containing children and adolescents together (SMD: $0 \cdot 12 ; 95 \%$ CI $-0 \cdot 01,0 \cdot 25 ; P_{\text {heterogeneity }}<0 \cdot 001$; $I^{2}=89.7 \%$ ). A small increase in mean BMI was observed in the subgroup analysis of studies including only adolescents aged 14 years or older (WMD: 0.58; 95\% CI 0.52, 0.64; $\left.P_{\text {heterogeneity }}<0 \cdot 001 ; I^{2}=98 \cdot 1 \%\right)$.

\section{A meta-analysis of healthy dietary patterns and cardiometabolic risk factors}

The consumption of large quantities of healthy foods was inversely associated with BMI and WC (Fig. 3). Compared with adolescents who consumed higher quantities of healthy foods, adolescents who had a low intake of these foods had a $0.41 \mathrm{~kg} / \mathrm{m}^{2}$ lower mean BMI (95\% CI -0.46, -0.36; $P_{\text {heterogeneity }}<0.001$; $\left.I^{2}=99 \cdot 2 \%\right)$ and a $0.43-\mathrm{cm}$ lower mean WC (95\% CI $-0.52,-0 \cdot 33$; $\left.P_{\text {heterogeneity }}<0.001 ; I^{2}=99 \cdot 3 \%\right)$. Similar to the results observed for unhealthy food patterns, the subgroup analysis of samples composed only of teenagers aged 14 years or older favoured an increase in mean WC (WMD: $-0.87 ; 95 \%$ CI $-1 \cdot 01,-0.73$; $\left.P_{\text {heterogeneity }}<0.001 ; I^{2}=99.5 \%\right)$ and maintenance of the mean BMI (SMD: $-0.41 ; 95 \%$ CI $-0.46,-0.36 ; \quad P_{\text {heterogeneity }}<0.001$; $\left.I^{2}=99 \cdot 2 \%\right)$ compared with studies including both children and adolescents.

Considering the reduced number of observations for each anthropometric measure, individual meta-regression models were constructed. Each model analysed the influence of the BMI (only for WC), the mean age and the sample size of the studies on the heterogeneity identified in the meta-analyses undertaken in this study. However, none of these variables explained the heterogeneity (results not shown). In addition, we undertook the sensibility analysis, excluding the studies with discrepant results, but the $I^{2}$ statistic was kept higher than $90 \%$ and the $P$ value was yet significant $(P<0001)$, indicating that the high heterogeneity identified in this meta-analysis is probably an intrinsic characteristic of the studies evaluating food pattern. Because only seven studies were included in this meta-analysis, Egger's test for publication bias could not be performed.

\section{Discussion}

Studies evaluating the association between dietary patterns and CMR factors were included in this systematic review and metaanalysis. The results revealed some evidence that unhealthy dietary patterns, assessed as higher quintiles/quartiles/tertiles or higher scores of factorial/cluster/PC analyses, were associated with higher mean values of WC, BMI, BP, lipid profile and blood glucose. The results of the association between healthy dietary patterns and the same risk factors were not statistically significant, as identified for unhealthy dietary patterns, and some healthy dietary patterns even showed the same associations with CMR factors as unhealthy dietary patterns. For example, the results of the meta-analysis suggested an 
(a)

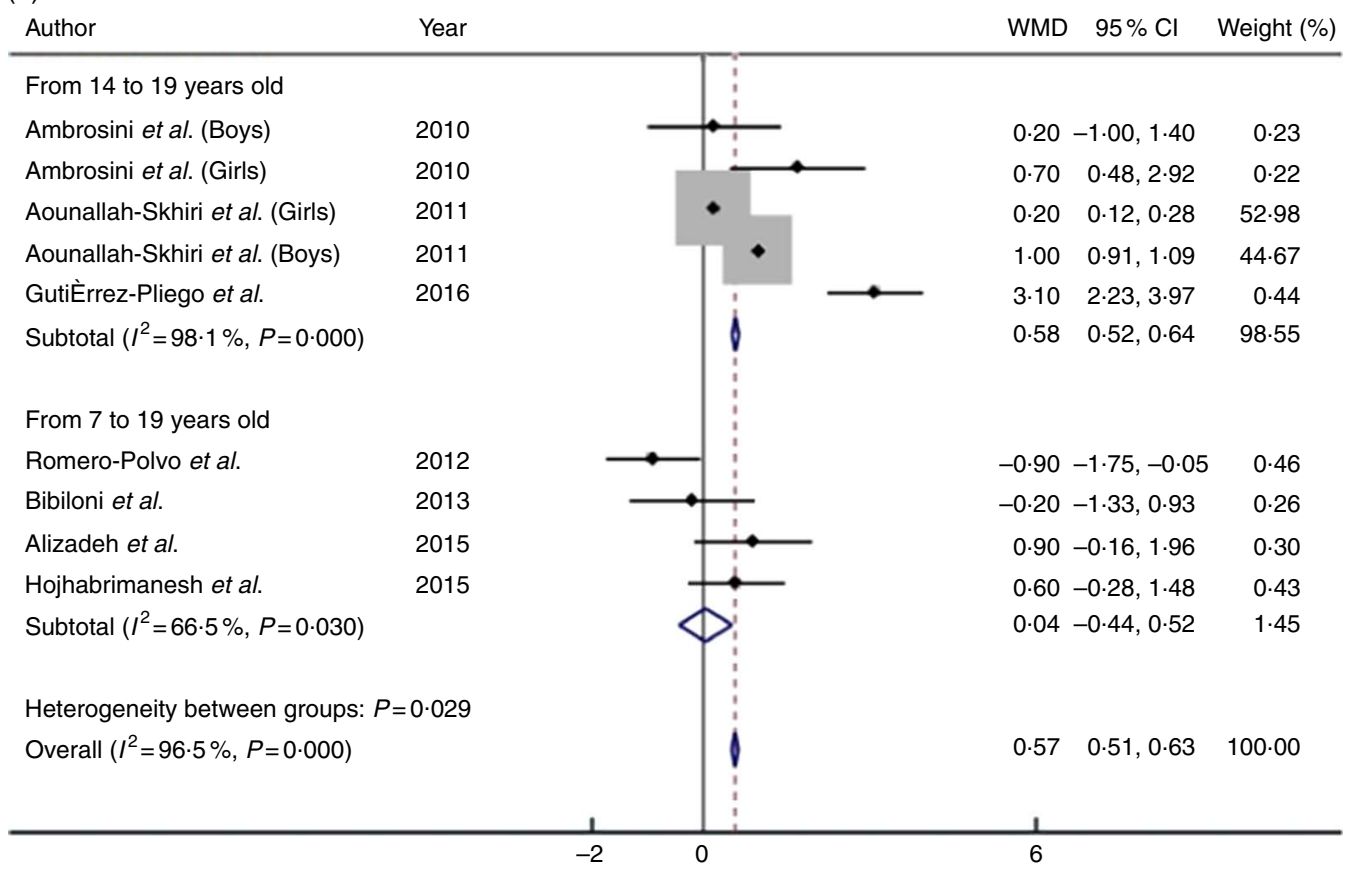

(b)

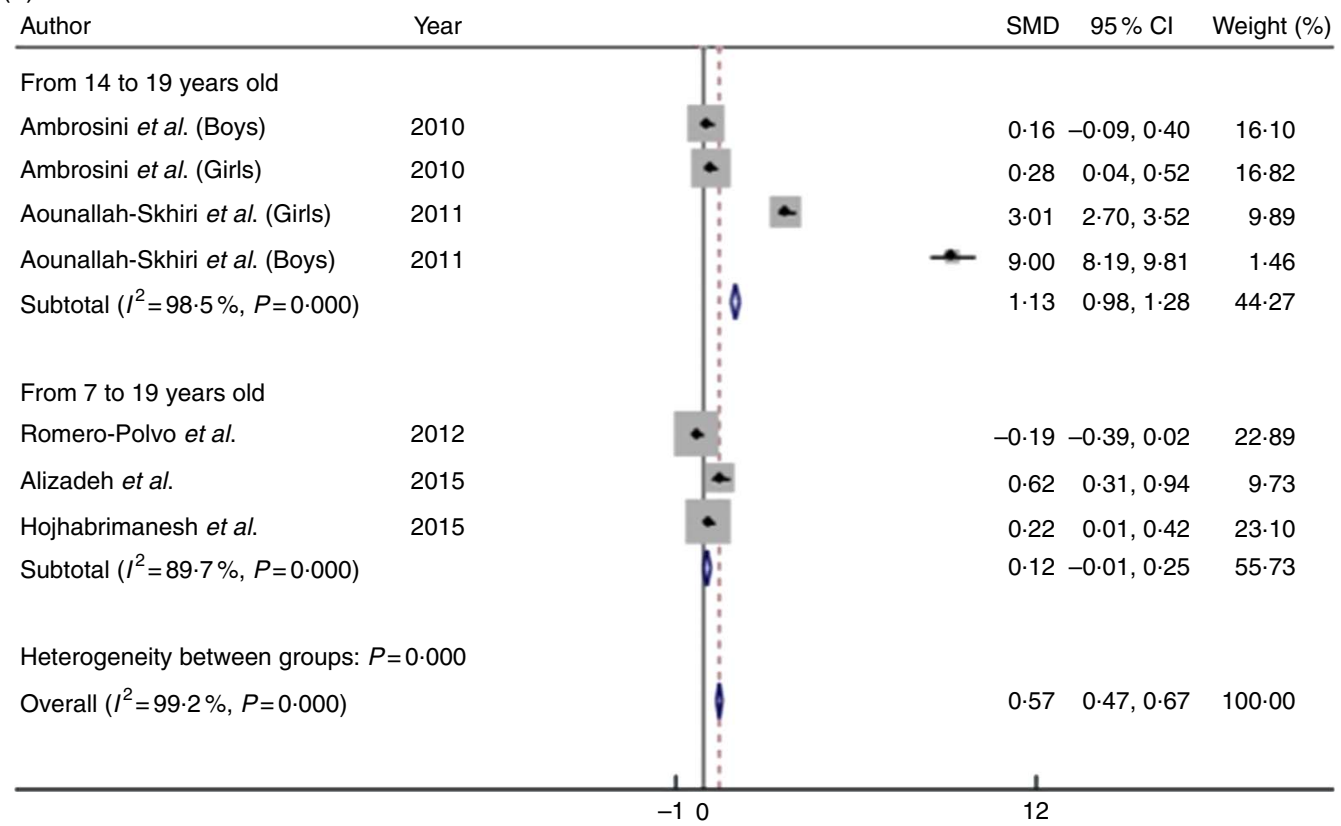

Fig. 2. Mean difference in BMI (a) and waist circumference (b) of high intake $v$. low intake of unhealthy food patterns among adolescents. WMD, weighted mean difference; SMD, standardised mean difference.

association between consuming large amounts of unhealthy diets and higher mean values of WC and BMI; this association increased in the subgroup analyses that included only adolescents. Elevated intake of healthier foods was associated with the highest mean WC and mean BMI, which conflicts with the protective effect that healthy dietary patterns are expected to have against CMR factors.

The studies included in this systematic review were mostly cross-sectional, applying a variety of different methodologies, dietary assessment methods (FFQ or 24-h dietary recall), statistical analyses to obtain dietary patterns and techniques or methods to measure outcomes and characterised by a range of limitations owing to adjusting for confounding variables. These characteristics emphasise the importance of cautiously interpreting the results of this study and reinforce the need to improve the quality of observational studies that involve the evaluation of food patterns in adolescents. In addition, the elevated heterogeneity observed in the results of the 
(a)

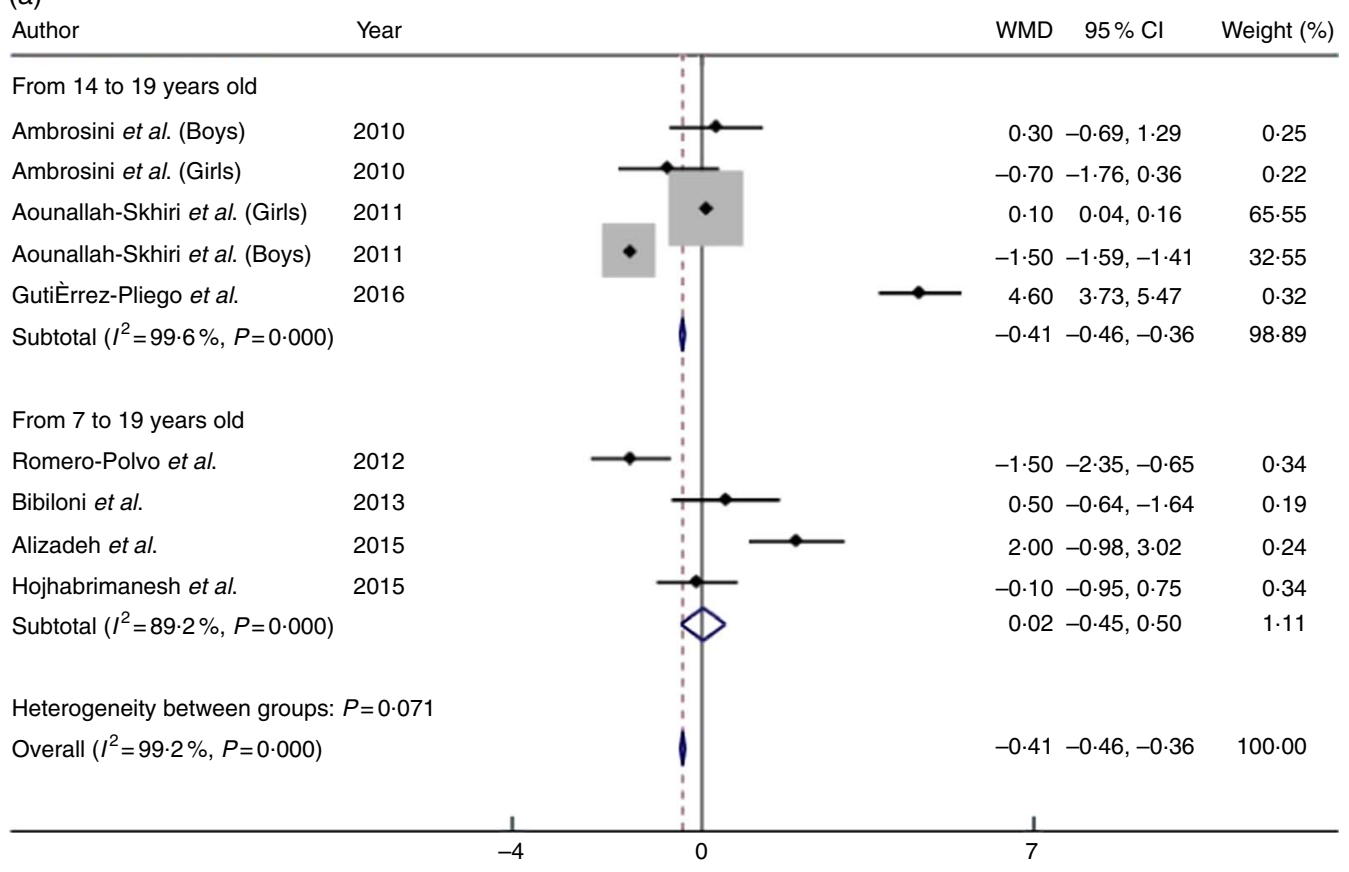

(b)

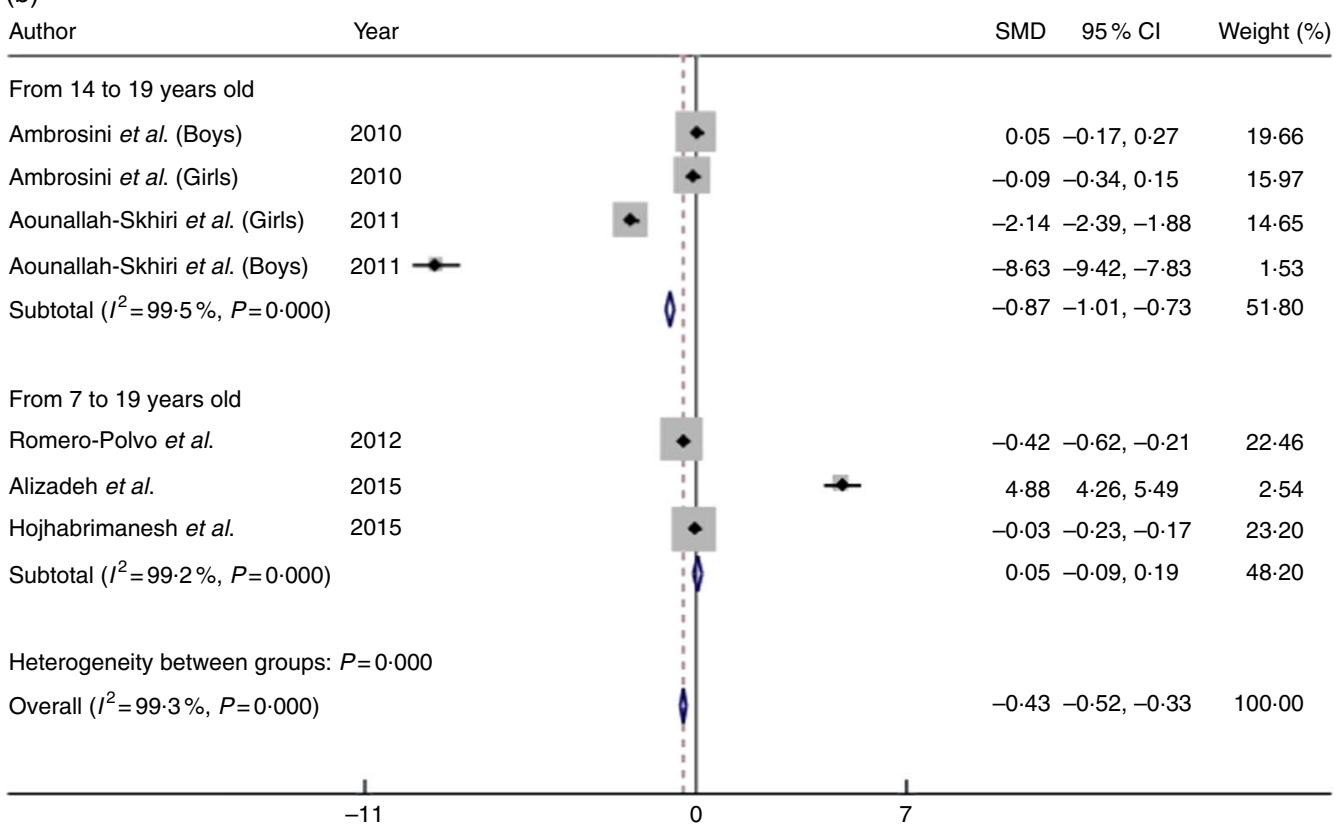

Fig. 3. Mean difference in BMI (a) and waist circumference (b) of low intake v. high intake of healthy food patterns among adolescents. WMD, weighted mean difference; SMD, standardised mean difference.

meta-analysis was considerable owing to the reduced number of studies presenting data for quintiles/quartiles/terciles. In addition, the methodological and analytical variation inherent to the food pattern complex variable can also contribute to the high heterogeneity. Then, considering that the exploration of heterogeneity using meta-regression, subgroup and sensitivity analysis was unlikely to explain the variation between the studies, caution should be applied when interpreting the summary effect measure calculated for this study.
Taking into consideration the study population, it is important to highlight that cardiometabolic markers are influenced by adolescence characteristics, such as hormonal changes during puberty and sexual maturation status, which may influence the association between diet and CMR factors ${ }^{(41,42)}$. Pubertal status promotes the occurrence of insulin resistance and influences lipid levels, body fat and lean mass distributions, which raise questions about using definitions for the metabolic syndrome and standardised cut-off points for health risk factors in children 
and adolescents that do not consider pubertal status ${ }^{(42,43)}$. The lack of a consensus for cut-off points to diagnose dyslipidaemia, hypertension, hyperglycaemia and overweight status results in the adoption of country-specific references for clinical studies. Therefore, the results of studies performed in different continents or countries may not be valid for comparison.

Interpretations and comparability of dietary patterns are challenging considering that adherence to a pattern can be influenced by a complex set of factors that include the socioeconomic status, family characteristics, environment, seasonality, culture and religious and traditional customs ${ }^{(44-48)}$. These characteristics are likely to produce different results, with patterns with diverse food content, making comparisons and interpretation of data across studies difficult. The evaluation and label assigned to each dietary pattern is defined by authors, considering their knowledge and expertise, which also may limit the comparability of studies.

Obtaining food consumption data also presents several forms of bias depending on the method that is used. Traditionally, information on food intake is obtained through 24-h recall or FFQ written. Such methods require extensive interview time, the information is influenced by interviewee's memory and cognition and the process may generate disinterest among participants, which is particularly critical for studies aimed at investigating the food consumption of children and adolescents $^{(49,50)}$. Therefore, dietetic risk analyses should be carefully planned by expert researchers and consider controlling for many variables to reduce confounding influence. In addition, estimating the relation between diet and disease can be enlarged when considering the food content and complex interactions among diet components; the intake of single nutrients never occurs in isolation, although the effects of nutrients are generally measured experimentally and not always confirmed in a human population ${ }^{(51,52)}$. Dietary pattern analyses provide diet information considering the entire scope of food content, diet composition and complex nutrient interactions and may explain the food-disease relationship better than investigating single nutrients or isolated foods ${ }^{(53,54)}$

The healthier food patterns observed in this study were mostly composed of legumes, vegetables, fruit, fish, low-fat dairy products, nuts, olive oil and others (Tables 2 and 3). These foods are frequently observed to be part of the Mediterranean diet, which has been shown to improve health and prevent health problems by its nutritional components of antioxidants, micronutrients, fibre, whole grains and monounsaturated and essential fatty acids ${ }^{(55,56)}$. The Mediterranean diet has also been successfully used as a non-medical treatment for obesity and other comorbidities and has had positive effects among intervention trials. Consequently, health societies widely recommend the Mediterranean diet to achieve healthier clinical parameters in the population ${ }^{(57-60)}$. This treatment, which is characteristic of healthy diets, may explain our findings in adolescents who had a higher intake of healthy foods and presented with higher mean BMI and WC, considering that some of them may be following dietetic recommendations directed by professionals to improve their health status. This is an important source of bias that has been observed in crosssectional studies (survival bias) ${ }^{(61)}$.
However, even given the territorial and cultural diversity of the included studies, we observed a similarity in food content in adolescents' unhealthy dietary patterns, including that the foods chosen by adolescents may be influenced by a globalised pattern, which may expose them to common dietetic risk factors and favours association among unhealthy diets and CMR factors $^{(9,14,62)}$. Foods that comprised unhealthy food patterns that were present in the selected studies were mainly represented by red and processed meat, confectionery and bakery items, full-fat dairy products, refined grains, desserts and candies. Unhealthy food patterns also included a reduced intake of vegetables and fruits (Tables 2 and 3). The combination of foods that have a high energetic density and a low nutritional quality is commonly observed in Western food habits and influences the label of some food patterns that describe a high $\mathrm{CMR}^{(63,64)}$. These food characteristics are frequently associated with undesirable health outcomes because of their elevated content of saturated and trans fatty acids, salt, high glycaemic index and low content of fibre and micronutrients ${ }^{(65,66)}$

In addition, the high energetic content observed in Western foods contributes to weight gain and central obesity, with strong evidence in adults and moderate evidence in children and adolescents $^{(67-71)}$.

BMI and WC present a clear relationship with other CMR factors $^{(72-75)}$, revealing that unhealthy eating habits may directly influence BP, insulin resistance and atherogenic lipid profile via nutrient metabolic repercussions and, secondarily, by contributing to an increase in weight and body circumference, which was observed in this study.

Nonetheless, this review is characterised by various limitations. Publication bias may be present, considering that unexpected or implausible associations between diet and CMR factors may not be published. Considering food intake analysis, random error is common and is attributed to the nature of the information obtained to test associations or to obtain dietary patterns. The methodologically distinct strategies and the crosssectional characteristics of many studies make it impossible to determine the causality of relationships of interest and, therefore, may affect the interpretation of the results.

In the review process, all methodology steps were taken into account to prevent biases. The research, data extraction and evaluation of bias were performed independently by the researchers. To obtain the maximum number of published papers in the field, six important data sources were used to search for relevant articles using MeSh terms exploding all trees. The reference lists of the retained articles and reviews were also evaluated to include additional studies.

This study found that adhering to an unhealthy dietary pattern appears to be associated with the worst values of CMR factors among adolescents. However, evidence of a protective effect of healthier dietary patterns in this group remains unclear. The meta-analysis results need to be cautiously interpreted considering the elevated heterogeneity; however, the high methodological quality applied in this study reinforces the validity of results. Considering the physiological changes associated with this phase of life, the lack of consensus among cut-off points for clinical parameters and the impact of food habits on health outcomes during early and adult life, further 
high-quality methodological research is warranted to estimate the potential impact of diet on CMR factors in adolescents. The publication of high-quality evidence in this field can strengthen clinical strategies to improve the health status of younger generations and to prevent undesirable outcomes during adulthood.

\section{Acknowledgements}

The authors thank the editor and anonymous reviewers for their constructive and valuable comments, which helped the authors improve the manuscript. We also thank the Fundação Oswaldo Cruz (Fiocruz) for providing EMBASE access.

This research received no specific grant from any funding agency, commercial or not-for-profit sectors.

C. d. M. C. and P. R. F. C. formulated the research question. J. C. D. P., P. R. F. C. and C. d. M. C. designed the study, conducted the study and analysed the data. V. A. d. O. Q. and L. P. M. d. O. evaluated the risk of bias. C. d. M. C. and A. M. O. A. discussed the results and wrote the paper. All of the authors contributed to the revision of the manuscript and read and approved the final version.

None of the authors has any conflicts of interest to declare.

\section{References}

1. Moschonis G, Tsoutsoulopoulou K, Efstathopoulou E, et al. (2015) Conceptual framework of a simplified multidimensional model presenting the environmental and personal determinants of cardiometabolic risk behaviors in childhood. Expert Rev Cardiovasc Ther 13, 673-692.

2. Eckel RH, Kahn R, Robertson RM, et al. (2006) Preventing cardiovascular disease and diabetes: a call to action from the American Diabetes Association and the American Heart Association. Circulation 113, 2943-2946.

3. NCD Risk Factor Collaboration (NCD-RisC) (2016) Trends in adult body-mass index in 200 countries from 1975 to 2014: a pooled analysis of 1698 population-based measurement studies with $19 \cdot 2$ million participants. Lancet 387, 1377-1396.

4. Janghorbani M, Salamat MR, Amini M, et al. (2017) Risk of diabetes according to the metabolic health status and degree of obesity. Diabetes Metab Syndr 11, Suppl. 1, S439-S444.

5. Mika A \& Sledzinski T (2017) Alterations of specific lipid groups in serum of obese humans: a review. Obes Rev 18, 247-272.

6. Cabandugama PK, Gardner MJ \& Sowers JR (2017) The renin angiotensin aldosterone system in obesity and hypertension: roles in the cardiorenal metabolic syndrome. Med Clin North Am 101, 129-137.

7. World Health Organization (2012) Population-Based Approaches to Childhood Obesity Prevention. Geneve: WHO.

8. World Health Organization (2000) Obesity: Preventing and Managing the Global Epidemic. WHO Technical Report Series. Geneva: WHO.

9. Lobstein T, Jackson-Leach R, Moodie ML, et al. (2015) Child and adolescent obesity: part of a bigger picture. Lancet $\mathbf{3 8 5}$, 2510-2520.

10. Agirbasli M, Tanrikulu AM \& Berenson GS (2016) Metabolic syndrome: bridging the gap from childhood to adulthood. Cardiovasc Ther 34, 30-36.

11. Abreu AP \& Kaiser UB (2016) Pubertal development and regulation. Lancet Diabetes Endocrinol 4, 254-264.

12. Hobbs M, Pearson N, Foster PJ, et al. (2015) Sedentary behaviour and diet across the lifespan: an updated systematic review. Br J Sports Med 49, 1179-1188.
13. Camhi SM \& Katzmarzyk PT (2010) Tracking of cardiometabolic risk factor clustering from childhood to adulthood. Int J Pediatr Obes 5, 122-129.

14. Funtikova AN, Navarro E, Bawaked RA, et al. (2015) Impact of diet on cardiometabolic health in children and adolescents. Nutr J 14, 118.

15. Rodríguez-Monforte M, Flores-Mateo G \& Sánchez E (2015) Dietary patterns and CVD: a systematic review and metaanalysis of observational studies. Br J Nutr 114, 1341-1359.

16. Li F, Hou LN, Chen W, et al. (2015) Associations of dietary patterns with the risk of all-cause, CVD and stroke mortality: a meta-analysis of prospective cohort studies. Br J Nutr 113, 16-24.

17. Clar C, Al-Khudairy L, Loveman E, et al. (2017) Low glycaemic index diets for the prevention of cardiovascular disease. Cochrane Database Syst Rev, issue 7, CD004467.

18. Silva DF, Lyra CeO \& Lima SC (2016) [Dietary habits of adolescents and associated cardiovascular risk factors: a systematic review]. Cien Saude Colet 21, 1181-1196.

19. Rocha NP, Milagres LC, Longo GZ, et al. (2017) Association between dietary pattern and cardiometabolic risk in children and adolescents: a systematic review. J Pediatr (Rio J) 93, 214-222.

20. Viswanathan M \& Berkman ND (2012) Development of the RTI item bank on risk of bias and precision of observational studies. J Clin Epidemiol 65, 163-178.

21. Higgins JPT \& Green S (editors) (2011) Cochrane Handbook for Systematic Reviews of Interventions Version 5.1.0, [updated March 2011]. The Cochrane Collaboration, 2011. www.handbook.cochrane.org.

22. Ambrosini GL, Huang RC, Mori TA, et al. (2010) Dietary patterns and markers for the metabolic syndrome in Australian adolescents. Nutr Metab Cardiovasc Dis 20, 274-283.

23. McNaughton SA, Ball K, Mishra GD, et al. (2008) Dietary patterns of adolescents and risk of obesity and hypertension. J Nutr 138, 364-370.

24. Ochoa-Avilés A, Verstraeten R, Lachat C, et al. (2014) Dietary intake practices associated with cardiovascular risk in urban and rural Ecuadorian adolescents: a cross-sectional study. BMC Public Health 14, 939.

25. Romero-Polvo A, Denova-Gutierrez E, Rivera-Paredez B, et al. (2012) Association between dietary patterns and insulin resistance in Mexican children and adolescents. Ann Nutr Metab 61, 142-150.

26. Ritchie LD, Spector P, Stevens MJ, et al. (2007) Dietary patterns in adolescence are related to adiposity in young adulthood in black and white females. J Nutr 137, 399-406.

27. Gutiérrez-Pliego LE, Del Socorro Camarillo-Romero E, Montenegro-Morales LP, et al. (2016) Dietary patterns associated with body mass index (BMI) and lifestyle in Mexican adolescents. BMC Public Health 16, 850.

28. Zamora-Gasga V M, Montalvo-González E, Loarca-Piña G F, et al. (2017) Dietary patterns, nutritional profile, and body mass index in Mexican schoolchildren: a cross-sectional study. Arch Latinoam Nutr 67, 6-14.

29. Alizadeh M, Didarloo A \& Esmaillzadeh A (2015) Dietary patterns of young females and their association with waist circumference as a health index in northwest of Iran, 2007. Iran Red Crescent Med J 17, e17594.

30. Bahreynian M, Paknahad Z \& Maracy MR (2013) Major dietary patterns and their associations with overweight and obesity among Iranian children. Int J Prev Med 4, 448-458.

31. Hojhabrimanesh A, Akhlaghi M, Rahmani E, et al. (2015) A Western dietary pattern is associated with higher blood pressure in Iranian adolescents. Eur J Nutr 56, 399-408.

32. Joung H, Hong S, Song Y, et al. (2012) Dietary patterns and metabolic syndrome risk factors among adolescents. Korean $J$ Pediatr 55, 128-135. 
33. Lee JW, Hwang J \& Cho HS (2007) Dietary patterns of children and adolescents analyzed from 2001 Korea National Health and Nutrition Survey. Nutr Res Pract 1, 84-88.

34. Shang X, Li Y, Liu A, et al. (2012) Dietary pattern and its association with the prevalence of obesity and related cardiometabolic risk factors among Chinese children. PLOS ONE 7, e43183.

35. Song $\mathrm{Y}$, Joung $\mathrm{H}$, Engelhardt $\mathrm{K}$, et al. (2005) Traditional v. modified dietary patterns and their influence on adolescents nutritional profile. Br J Nutr 93, 943-949.

36. Weng TT, Hao JH, Qian QW, et al. (2012) Is there any relationship between dietary patterns and depression and anxiety in Chinese adolescents? Public Health Nutr 15, 673-682.

37. Del Mar Bibiloni M, Maffeis C, Llompart I, et al. (2013) Dietary factors associated with subclinical inflammation among girls. Eur J Clin Nutr 67, 1264-1270.

38. Monjardino T, Lucas R, Ramos E, et al. (2014) Associations between a posteriori defined dietary patterns and bone mineral density in adolescents. Eur J Nutr 54, 273-282.

39. Przysławski J, Stelmach M, Grygiel-Górniak B, et al. (2011) Dietary habits and nutritional status of female adolescents from the great Poland region. Pol J Food Nutr Sci 61, 73-78.

40. Aounallah-Skhiri H, Traissac P, El Ati J, et al. (2011) Nutrition transition among adolescents of a south-Mediterranean country: dietary patterns, association with socio-economic factors, overweight and blood pressure. A cross-sectional study in Tunisia. Nutr J 10, 38.

41. Eissa MA, Mihalopoulos NL, Holubkov R, et al. (2016) Changes in fasting lipids during puberty. J Pediatr 170, 199-205.

42. Reinehr T (2016) Metabolic syndrome in children and adolescents: a critical approach considering the interaction between pubertal stage and insulin resistance. Curr Diab Rep 16, 8.

43. Moon S, Park JS \& Ahn Y (2017) The cut-off values of triglycerides and glucose index for Metabolic syndrome in American and Korean adolescents. J Korean Med Sci 32, 427-433.

44. Pollard CM, Landrigan TJ, Ellies PL, et al. (2014) Geographic factors as determinants of food security: a Western Australian food pricing and quality study. Asia Pac J Clin Nutr 23, 703-713.

45. Chi DL, Luu M \& Chu F (2017) A scoping review of epidemiologic risk factors for pediatric obesity: Implications for future childhood obesity and dental caries prevention research. J Public Health Dent 77, Suppl. 1, S8-S31.

46. Manyanga T, Tremblay MS, Chaput JP, et al. (2017) Socioeconomic status and dietary patterns in children from around the world: different associations by levels of country human development? BMC Public Health 17, 457.

47. Giskes K, van Lenthe F, Avendano-Pabon M, et al. (2011) A systematic review of environmental factors and obesogenic dietary intakes among adults: are we getting closer to understanding obesogenic environments? Obes Rev 12, e95-e106.

48. Fernández-Alvira JM, Börnhorst C, Bammann K, et al. (2015) Prospective associations between socio-economic status and dietary patterns in European children: The Identification and Prevention of Dietary- And Lifestyle-induced Health Effects in Children and Infants (IDEFICS) study. Br J Nutr 113, 517-525.

49. Six BL, Schap TE, Zhu FM, et al. (2010) Evidence-based development of a mobile telephone food record. J Am Diet Assoc 110, 74-79.

50. Probst Y, Nguyen DT, Tran MK, et al. (2015) Dietary assessment on a mobile phone using image processing and pattern recognition techniques: algorithm design and system prototyping. Nutrients 7, 6128-6138.

51. Stradling C, Hamid M, Taheri S, et al. (2014) A review of dietary influences on cardiovascular health: part 2: dietary patterns. Cardiovasc Hematol Disord Drug Targets 14, 50-63.

52. Bakolis I, Burney P \& Hooper R (2014) Principal components analysis of diet and alternatives for identifying the combination of foods that are associated with the risk of disease: a simulation study. Br J Nutr 112, 61-69.

53. Castro MA, Baltar VT, Selem SS, et al. (2015) Empirically derived dietary patterns: interpretability and construct validity according to different factor rotation methods. Cad Saude Publica 31, 298-310.

54. Cunha DB, Almeida RM \& Pereira RA (2010) A comparison of three statistical methods applied in the identification of eating patterns. Cad Saude Publica 26, 2138-2148.

55. Badimon L, Chagas P \& Chiva-Blanch G (2017) Diet and cardiovascular disease: effects of foods and nutrients in classical and emerging cardiovascular risk factors. Curr Med Chem (epublication ahead of print version 27 April 2017)

56. Shen J, Wilmot KA, Ghasemzadeh N, et al. (2015) Mediterranean dietary patterns and cardiovascular health. Annu Rev Nutr 35, 425-449.

57. Sacks FM, Lichtenstein AH, Wu JHY, et al. (2017) Dietary fats and cardiovascular disease: a presidential advisory from the American Heart Association. Circulation 136, e1-e23.

58. American Diabetes Association (2017) 4. Lifestyle management. . Diabetes Care 40, S33-S43.

59. Garvey WT, Mechanick JI, Brett EM, et al. (2016) American Association of Clinical Endocrinologists and American College of Endocrinology Comprehensive Clinical Practice Guidelines for Medical Care of Patients with Obesity. Endocr Pract 22, Suppl. 3, 1-203.

60. Piepoli MF, Hoes AW, Agewall S, et al. (2016) 2016 European Guidelines on cardiovascular disease prevention in clinical practice: The Sixth Joint Task Force of the European Society of Cardiology and Other Societies on Cardiovascular Disease Prevention in Clinical Practice (constituted by representatives of 10 societies and by invited experts). Developed with the special contribution of the European Association for Cardiovascular Prevention \& Rehabilitation (EACPR). Eur Heart J37, 2315-2381.

61. Rothman KJ, Greenland S \& Lash TL (2008) Modern Epidemiology, 3rd ed. Philadelphia, PA: Lippincott Williams \& Wilkins.

62. Oberlander L, Disdier AC \& Etilé F (2017) Globalisation and national trends in nutrition and health: a grouped fixed-effects approach to intercountry heterogeneity. Health Econ 26, $1146-1161$

63. Anand SS, Hawkes C, de Souza RJ, et al. (2015) Food consumption and its impact on cardiovascular disease: importance of solutions focused on the globalized food system: a report from the Workshop Convened by the World Heart Federation. J Am Coll Cardiol 66, 1590-1614.

64. Sherzai A, Heim LT, Boothby C, et al. (2012) Stroke, food groups, and dietary patterns: a systematic review. Nutr Rev 70, 423-435.

65. Angelieri CT, Barros CR, Siqueira-Catania A, et al. (2012) Trans fatty acid intake is associated with insulin sensitivity but independently of inflammation. Braz J Med Biol Res 45, 625-631.

66. Li Y, Hruby A, Bernstein AM, et al. (2015) Saturated fats compared with unsaturated fats and sources of carbohydrates in relation to risk of coronary heart disease: a prospective cohort study. J Am Coll Cardiol 66, 1538-1548.

67. Nagao M, Asai A, Sugihara H, et al. (2015) Fat intake and the development of type 2 diabetes. Endocr J 62, 561-572.

68. Pérez-Escamilla R, Obbagy JE, Altman JM, et al. (2012) Dietary energy density and body weight in adults and children: a systematic review. I Acad Nutr Diet 112, 671-684.

69. Hooper L, Abdelhamid A, Bunn D, et al. (2015) Effects of total fat intake on body weight. Cochrane Database Syst Rev, issue 8, CD011834. 
70. Pate RR, O'Neill JR, Liese AD, et al. (2013) Factors associated with development of excessive fatness in children and adolescents: a review of prospective studies. Obes Rev 14, 645-658.

71. Howe AS, Black KE, Wong JE, et al. (2013) Dieting status influences associations between dietary patterns and body composition in adolescents: a cross-sectional study. Nutr J 12, 51.

72. Ma C, Wang R, Liu Y, et al. (2016) Performance of obesity indices for screening elevated blood pressure in pediatric population: systematic review and meta-analysis. Medicine (Baltimore) 95, e4811.
73. Cheung EL, Bell CS, Samuel JP, et al. (2017) Race and obesity in adolescent hypertension. Pediatrics 139, e20161433.

74. Subasinghe AK, Wark JD, Gorelik A, et al. (2017) The association between inflammation, obesity and elevated blood pressure in 16-25-year-old females. J Hum Hypertens 31 , 580-584.

75. Dong B, Wang Z, Arnold LW, et al. (2016) Role of waist measures in addition to body mass index to assess the hypertension risk in children. Blood Press 25 , 344-350. 